\title{
Observation of the full 12-hour-long transit of the exoplanet HD 80606b^
}

\section{Warm-Spitzer photometry and SOPHIE spectroscopy}

\author{
G. Hébrard ${ }^{1}$, J.-M. Désert ${ }^{2,1}$, R. F. Díaz ${ }^{1}$, I. Boisse ${ }^{1}$, F. Bouchy ${ }^{1,3}$, A. Lecavelier des Etangs ${ }^{1}$, C. Moutou ${ }^{4}$,
} D. Ehrenreich ${ }^{5}$, L. Arnold ${ }^{3}$, X. Bonfils ${ }^{5,7}$, X. Delfosse ${ }^{5}$, M. Desort ${ }^{5}$, A. Eggenberger ${ }^{5}$, T. Forveille ${ }^{5}$, J. Gregorio ${ }^{6}$, A.-M. Lagrange ${ }^{5}$, C. Lovis ${ }^{7}$, F. Pepe ${ }^{7}$, C. Perrier ${ }^{5}$, F. Pont ${ }^{8}$, D. Queloz ${ }^{7}$, A. Santerne ${ }^{4}$, N. C. Santos ${ }^{9}$, D. Ségransan ${ }^{7}$, D. K. Sing ${ }^{8}$, S. Udry ${ }^{7}$, and A. Vidal-Madjar ${ }^{1}$

\footnotetext{
1 Institut d'Astrophysique de Paris, UMR7095 CNRS, Université Pierre \& Marie Curie, 98bis boulevard Arago, 75014 Paris, France e-mail: hebrard@iap.fr

2 Harvard-Smithsonian Center for Astrophysics, 60 Garden Street, Cambridge, MA 02138, USA

Observatoire de Haute-Provence, CNRS/OAMP, 04870 Saint-Michel-l'Observatoire, France

${ }^{4}$ Laboratoire d'Astrophysique de Marseille, Univ. de Provence, CNRS (UMR6110), 38 rue F. Joliot Curie, 13388 Marseille, France

5 Laboratoire d'Astrophysique de Grenoble, Université Joseph-Fourier, CNRS (UMR5571), BP 53, 38041 Grenoble Cedex 9, France

${ }^{6}$ CROW-observatory Portalegre, Portugal, and Atalaia.org group, Portugal

7 Observatoire de Genève, Université de Genève, 51 chemin des Maillettes, 1290 Sauverny, Switzerland

8 School of Physics, University of Exeter, Exeter, EX4 4QL, UK

9 Centro de Astrofísica, Universidade do Porto, Rua das Estrelas, 4150-762 Porto, Portugal
}

Received 26 February 2010 / Accepted 5 April 2010

\section{ABSTRACT}

\begin{abstract}
We present new observations of a transit of the 111.4-day-period exoplanet HD 80606b. Due to this long orbital period and to the orientation of the eccentric orbit ( $e=0.9$ ), HD 80606b's transits last for about 12 hours. This makes the observation of a full transit practically impossible from a given ground-based observatory. With the Spitzer Space Telescope and its IRAC camera on the postcryogenic mission, we performed a 19-h photometric observation of HD 80606 that covers the full 2010 January 13-14 transit as well as off-transit references immediately before and after the event. We complement these photometric data by new spectroscopic observations that we simultaneously performed with SOPHIE at the Haute-Provence Observatory. This provides radial velocity measurements of the first half of the transit that was previously uncovered with spectroscopy. This new dataset allows the parameters of this singular planetary system to be significantly refined. We obtained a planet-to-star radius ratio $R_{\mathrm{p}} / R_{*}=0.1001 \pm 0.0006$ that is more accurate but slightly lower than the one measured from previous ground observations in the optical. We found no astrophysical interpretations able to explain this difference between optical and infrared radii; we rather favor underestimated systematic uncertainties, maybe in the ground-based composite light curve. We detected a feature in the Spitzer light curve that could be due to a stellar spot. We also found a transit timing about 20 minutes earlier than the ephemeris prediction; this could be caused by actual transit-timing variations due to an additional body in the system, or again by underestimated systematic uncertainties. The actual angle between the spin-axis of HD 80606 and the normal to the planetary orbital plane is found to be near $40^{\circ}$ thanks to the fit of the Rossiter-McLaughlin anomaly, with a sky-projected value $\lambda=42^{\circ} \pm 8^{\circ}$. This allows scenarios with aligned spin-orbit to be definitively rejected. Over the twenty planetary systems with measured spin-orbit angles, a few are misaligned; this is probably the signature of two different evolution scenarios for misaligned and aligned systems, depending whether or not they experienced gravitational interaction with a third body. As in the case of HD 80606, most of the planetary systems including a massive planet are tilted; this could be the signature of a separate evolution scenario for massive planets compared with Jupiter-mass planets.
\end{abstract}

Key words. techniques: radial velocities - techniques: photometric - stars: individual: HD 80606

\section{Introduction}

Among the more than 400 extrasolar planets that have been found so far, the giant planet orbiting the G5 star HD 80606 is certainly a unique case. Its eccentricity is particularly

* Based on observations collected with the SOPHIE spectrograph on the 1.93-m telescope at the Observatoire de Haute-Provence (CNRS), France, and with the Spitzer Space Telescope, which is operated by the Jet Propulsion Laboratory, California Institute of Technology under a contract with NASA. Radial velocity and photometry tables are available in electronic form at the CDS via anonymous ftp to

cdsarc.u-strasbg.fr $(130.79 .128 .5)$ or via

http://cdsweb.u-strasbg.fr/cgi-bin/qcat?J/A+A/516/A95 high: $e=0.93$. Only one known planet possibly has a higher eccentricity, namely HD 20782b; however its high $e$-value is still to be confirmed as it rests on one measurement only (O'Toole et al. 2009). The comet-like orbit of HD 80606b was well established in its discovery paper by Naef et al. (2001) and has been largely confirmed by subsequent observations. Together with its 111.4-day period, the high eccentricity of HD 80606b put it on an extreme orbit: during its revolution, the planet experiences the strongly irradiated regime of a "hot Jupiter" at periastron (0.03 AU), and milder conditions at apoastron (0.87 AU) around which it spends most of its time, and where the planet approaches the inner boundary of the habitable zone (see Fig. 1). 


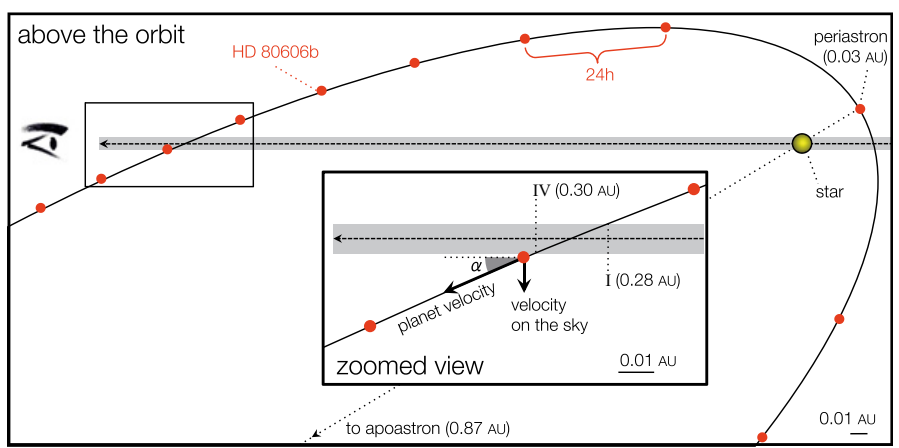

Fig. 1. Schematic view of the HD 80606 system. The red dots show the positions of the planet each $24 \mathrm{~h}$.

As long as the inclination $i$ of the orbit with the line of sight was unknown, those parameters originally implied a probability of $1 \%$ for the planet to be transiting. In spite of this tenuous probability, an amazing chance makes the planet HD 80606b actually transits its parent star, as seen from Earth, every 111.4 days. This is particularly advantageous as numerous crucial studies can be performed with photometry or spectroscopy when a planet passes in front its parent star (planetary transits) or behind it (planetary eclipses), especially in this case where the host star is bright $(V=9.1)$ and nearby $(d=60 \mathrm{pc})$. The fortunate transiting nature of HD 80606b was established in 2009 February from the detection of a transit reported from ground observations, independently by Moutou et al. (2009) from photometric and spectroscopic data, and by Garcia-Melendo \& McCullough (2009) and Fossey et al. (2009) from photometric measurements. Additional observations of transits were later reported by Winn et al. (2009a) and Hidas et al. (2010). The 2009 February observations followed the planetary eclipse discovery reported a few months before by Laughlin et al. (2009) from Spitzer photometric observations at $8 \mu \mathrm{m}$ during a $30-\mathrm{h}$ interval around the periastron. Among the known transiting planets, HD 80606b has the longest period and the most eccentric orbit. The second most extreme transiting planet is HD 17156b $(P=21.2$ days and $e=0.67)$. Furthermore, HD 80606b is also the most distant planet from its parent stars when it transits: 0.3 AU compared with $0.05 \mathrm{AU}$ or less for all other known cases ${ }^{1}$.

In addition to the photometric detection of the HD 80606b's transit, Moutou et al. (2009) presented its spectroscopic detection using the Rossiter-McLaughlin effect, measured with the SOPHIE spectrograph from radial velocities at the HauteProvence Observatory (OHP), France. The Rossiter-McLaughlin effect is an apparent distortion of the stellar line profiles due to the transit of the planet in front of the rotating star. From the SOPHIE measurements, Moutou et al. (2009) have shown the first evidence for a spin-orbit misalignment, i.e. the orbital plane of the planet HD 80606b is not perpendicular to the spinaxis of its host star. Using additional photometric data of the 2009 February event that allowed a better constraint on the transit duration together with a combined analysis of the whole dataset, Pont et al. (2009) refined the parameters of the system. They confirmed the spin-orbit misalignment from the RossiterMcLaughlin distortion detected with SOPHIE and provided a measurement of the sky-projected angle between the planetary orbital axis and the stellar rotation axis: $\lambda \sim 50^{\circ}$, with the confidence interval $\left[14^{\circ}-111^{\circ}\right]$ - see also Gillon (2009). Thanks to new photometric and spectroscopic observations of

\footnotetext{
1 See also the 95-day-period transiting exoplanet CoRoT-9b announced after the submission of the present paper (Deeg et al. 2010).
}

the 2009 June transit, Winn et al. (2009a) thereafter reduced the confidence interval even more to $\left[32^{\circ}-87^{\circ}\right]$. Thus the spinorbit misalignment of the HD 80606 system is now well established. The star HD 80606 is the component of a wide binary with HD 80607; the projected separation of the system is about $1000 \mathrm{AU}$. The peculiar orbit of HD 80606b could thus result from Kozai mechanism and tidal dissipation (see e.g., Wu \& Murray 2003), which can pump the eccentricity and the inclination.

Due to the long orbital period and to the orientation of the eccentric orbit, the duration of the transit of HD 80606b is about $12 \mathrm{~h}$. This should be compared to the transit duration of other known transiting exoplanets, which typically lasts less than five hours. The transit duration of HD 80606b is even longer than transits of Mercury or Venus through the Solar disk as seen from the Earth. It is thus practically impossible that a full transit of HD 80606b matches the duration of an observation night from ground. In addition, data secured before and after the transit are mandatory to obtain an accurate transit light curve, so the full sequence for HD 80606 lasts longer than a night for ground observations. Observing an entire transit of this exoplanet is thereby challenging, and only portions of a transit could be observed from a given ground-based telescope. This was the case of all the observation campaigns reported above which covered only fractions of transits. These fragmented observations induce significant uncertainties in the parameters derived from their fit.

We present here the first full photometric observation of a transit of HD 80606b. We secured it on 2010 January 1314 with the Spitzer space observatory using the IRAC camera at $4.5 \mu \mathrm{m}$ in post-cryogenic mission. Thanks to its Earthtrailing heliocentric orbit (Scoupe et al. 2006), Spitzer allowed us to continuously observe during $19 \mathrm{~h}$, enabling the coverage of the whole 12-hour-long transit, as well as off-transit references immediately before and after the event. We complement this photometric data by new spectroscopic observations that we simultaneously performed with SOPHIE at OHP. This provides radial velocity measurements of the first half of the transit, a part that was up to now not covered by spectroscopy. Indeed, observing the full 12-hour-long transit is even more difficult in spectroscopy than in photometry, as the amplitude of the RossiterMcLaughlin for HD 80606 is about $10 \mathrm{~m} \mathrm{~s}^{-1}$, whereas northern instruments able to measure radial velocities with the required accuracy are scarce. We also performed a ground-based photometric monitoring of HD 80606 during 2010 January. All together, the data of this observational campaign allow the parameters of this planetary system to be additionally refined.

The observations and data reduction are presented in Sects. 2 and 3 for Spitzer and SOPHIE respectively. The ground-based photometry is presented in Sect. 4 . The analyses and the results are presented in Sect. 5 before the discussion and conclusion in Sects. 6 and 7.

\section{Spitzer photometry}

\subsection{Observations}

We obtained Spitzer Director's Discretionary Time (DDT program \#540) to observe the 2010 January transit of HD 80606b. This transit was the first observable with Spitzer since the discovery of the transiting nature of HD $80606 \mathrm{~b}$ in 2009 February. As Spitzer has exhausted its cryogen of liquid coolant on 2009 May 15, our observations were performed during the first months of the Spitzer's warm mission. Only the first two infrared channels of the IRAC camera (Fazio et al. 2004) are available in 
the post-cryogenic Spitzer. They are centered at 3.6 and $4.5 \mu \mathrm{m}$ and cannot be observed simultaneously. We chose to observe only in one of the two channels to avoid repointing the telescope during the transit. This reduced overheads and ensured that the target remained on the same part of the detector during the full observation sequence. We opted for channel 2 at $4.5 \mu \mathrm{m}$ because it has the lowest noise properties. This wavelength also has a smaller limb-darkening effect for the star. We did not use pointing dithering, here again to maintain the target as much as possible in the same location on the detector to reduce systematic effects due to imperfect flat-field corrections and intra-pixel sensitivity variations.

We used the Subarray mode of IRAC, which is well adapted for bright targets. Only a $32 \times 32$-pixel part of the detector is used in this mode; this covers a small $38 \times 38 \operatorname{arcsec}^{2}$ field of view (pixel size of 1.2 arcsec), compared to the IRAC stellar mode that uses the full $256 \times 256$-pixel field. As the stellar companion HD 80607 is located only 17 arcsec to the East of HD 80606, putting the two targets on this small field of view would have implied that their point-spread functions (PSF) fell near the edges of the detector. This configuration is risky for accurate photometry. We preferred to let HD 80607 out of the field of view to maintain HD 80606 on the detector, far from its edges. So we chose to put our target at the default pointing position in the center of the subarray field of view. This position is not on nor right next to any known hot pixels.

The observations were secured between 2010 January 13 at $18 \mathrm{~h}$ and January 14 at $13 \mathrm{~h}$ (UT). We acquired 2488 consecutive images during a total of 19 hours. Each image was split up into 64 frames of 0.36 seconds exposure each, taken back-to-back. We obtained a total of 159232 frames during 15.9-hours effective integration time with an overhead of two seconds between each images (15\% overheads in total). This high efficiency is reached thanks to the use of the subarray mode and could not be achieved in stellar mode for bright targets requiring short exposure times. With a flux density of $\sim 200 \mathrm{mJy}$ at $4.5 \mu \mathrm{m}$ for HD 80606, the frame exposure time of $0.36 \mathrm{sec}$ clearly avoids saturation of the pixels. The intensities recorded in the brightest central pixels are around 10000 electrons.

\subsection{Data reduction}

We used the BCD files (Basic Calibrated Data) of each of the 159232 frames as they are produced by the Spitzer/IRAC pipeline. They include corrections for dark current, flat fielding, pixels non-linearity, and conversion to flux units. To extract the light curve, we used tools and methods we developed in Désert et al. $(2009,2010)$. We found the center of the PSF of the star to a precision of 0.01 pixel through the DAOPHOT-type Photometry Procedures, GCNTRD, from the IDL Astronomy Library ${ }^{2}$, which computes the stellar centroid by Gaussian fitting. We used the APER routine to extract the raw flux of HD 80606 on each frame from the computation of a simple aperture photometry with a radius of 4.0 pixels, which optimized the signal-to-noise ratio of the transit light curve. The flux integrated on these 50 pixels is $\sim 52500$ electrons. It was corrected from the background level of $14.40 \pm 0.05$ electrons/pixel that were estimated from a sky annulus with radii of 9 to 12 pixels. The centroid of HD 80607 is located outside the field of view, but a small contribution of its flux is detected on an edge of the detector. When estimating the background level, we took care to use only pixels where the HD 80607's flux is negligible. It is also negligible by

${ }^{2}$ http://idlastro.gsfc.nasa.gov/homepage.html comparison to the background level in the 4-pixel radius we used for the HD 80606 photometry. Finally, the uncertainty in the background level is negligible for all the results presented in this paper.

The Spitzer/IRAC photometry is known to be systematically affected by the so-called pixel-phase effect. This effect produces an oscillation of the measured raw light curve due to the Spitzer telescope jitter and the intra-pixel sensitivity variations on the IRAC detector (see e.g., Charbonneau et al. 2005; Reach et al. 2006; Morales-Calderón et al. 2006; Ehrenreich et al. 2007; Désert et al. 2009, 2010). Measurements of the centroid position of the target on the detector and its variations could be used to de-correlate the pixel-phase effect on the light curve. We used here the method presented in Désert et al. (2009); it has the form $F_{\text {corr }}=F\left[1+K_{1}\left(x-x_{0}\right)+K_{2}\left(x-x_{0}\right)^{2}+K_{3}\left(y-y_{0}\right)+K_{4}\left(y-y_{0}\right)^{2}+\right.$ $\left.K_{5}\left(x-x_{0}\right)\left(y-y_{0}\right)\right]$, where $F$ and $F_{\text {corr }}$ are the fluxes of the star before and after the pixel-phase effect correction, and $\left(x-x_{0}\right)$ and $\left(y-y_{0}\right)$ are the position in the pixels of the source centroid on the detector with respect to the pixel pointing position, located on $\left[x_{0}, y_{0}\right]$. Our determination of the centroid position shows a \pm 0.05 -pixel oscillation with a period of $\sim 70 \mathrm{~min}$ and a linear drift during the 19-h sequence of 0.1 and 0.2 pixel in the $x$ - and $y$-direction, respectively.

In addition to the 64 frames of the image \# 873 that are corrupted, we iteratively selected and trimmed 1037 outliers by comparison to the fit of the light curve with a transit model. Frames were considered as outliers when they were above 10 to $3 \sigma$, this value was reduced by $0.1-\sigma$ steps at each iterations. We binned the obtained transit light curve by a factor of five in order to obtain a better computing efficiency without losing information for the pixel phase effect. Most of the results presented below were obtained with the binned transit light curve.

We tested several radii for the aperture photometry, several areas for the background measurement, several procedures for the centroid determination and the outliers rejections. The adopted procedure reported above is the one producing the smallest errors, but all of them produced similar results.

The upper panel of Fig. 2 shows the raw Spitzer light curve of HD 80606 after this extraction. It clearly shows flux variations with a period of $\sim 70 \mathrm{~min}$ that are due to the pixel-phase effect and telescope jitter at this period. Its peak-to-peak amplitude represents $\sim 1 \%$ of the flux, which is larger than the effect seen in the channel 2 of IRAC during the cryogenic Spitzer. Additional variations at higher frequency and with a lower amplitude than the one of the 70-min oscillation were seen as well as we fitted each individual frame. They could be due to short-term jitter of the satellite, which are apparently not periodic. Filtering these high-frequency variations does not significantly change our results. Finally, a slope in the out-of-transit baseline is also seen. This detector ramp was observed in channels 3 and 4 in precryogenic Spitzer, but was not as significant in channel 2 at that time (see e.g., Deming et al. 2005; Knutson et al. 2007; Désert et al. 2009, 2010). We discarded the first thousands of frames that were the most affected by the ramp effect (we tested several limits; see Sect. 5.1.4) then normalized the light-curve using a time-dependent function with the form $F_{\text {baseline }}=A_{0}+A_{1} \times t$, where $F_{\text {baseline }}$ is the target flux out of transit and $t$ is the time. We also tried higher-degree polynomials and logarithmic baselines; this did not significantly improve the fit. The linear correction we adopted is not perfect; remaining uncertainties in the actual shape of the baseline introduced errors in the system parameters derived from the fit (see Sect. 5.1.4). The baseline and pixel-phase-effect parameters were fitted simultaneously with the transit-related parameters (see Sec. 5.1.3); this allowed these 


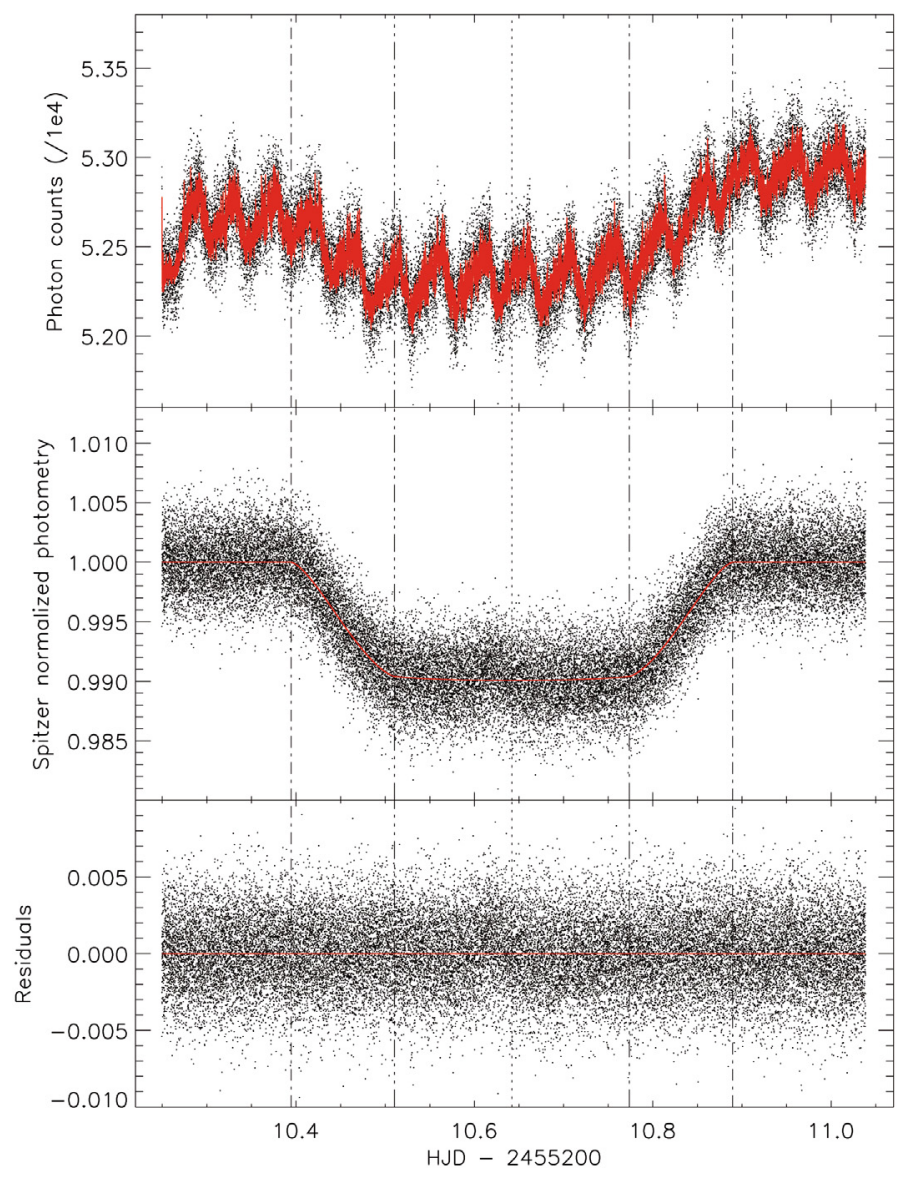

Fig. 2. Spitzer photometry of HD 80606. From top to bottom, the three panels show the raw photometry, the photometry after correction of the pixel-phase and ramp effects, and the residuals respectively. The black points are the measurements (binned per 5 frames) and the red solid lines are the fits. The vertical dotted lines show the mid-transit, the vertical dot-dashed lines show the first and fourth contacts, and the vertical dot-dot-dashed lines show the second and third contacts.

effects and their uncertainties to be taken into account in the transit parameters determination. The middle panel of Fig. 2 shows the light curve after removal of these instrumental effects and the lower panel shows the residuals to the fit.

\section{SOPHIE radial velocities}

\subsection{Observations}

The first half of the 2010 January transit of HD 80606b was visible from Europe, so we managed to observe it with the SOPHIE spectrograph at the 1.93-m telescope of the HauteProvence Observatory in South of France. SOPHIE is a crossdispersed, environmentally stabilized echelle spectrograph dedicated to high-precision radial velocity measurements (Perruchot et al. 2008, Bouchy et al. 2009). Since the discovery of the transit in 2009 February, this was the first time that this part of the transit was observable from an observatory with high-precision spectroscopic capabilities. Unfortunately, the 1.93-m telescope started a technical break for maintenance and upgrades in 2009 November that was extended up to 2010 February. Due to the importance of this event, some observations could nevertheless be performed thanks to the support of the OHP staff, wich was mandatory due to the ongoing works on the telescope.
The observations were secured as part of the second subprogram of the SOPHIE Consortium (Bouchy et al. 2009; Hébrard et al. 2010b). The night of the transit, 2010 January 13, observations could start just before $23 \mathrm{~h}$ (UT) after technical issues were solved and clouds disappeared, and had to be stopped $4.5 \mathrm{~h}$ later due to cloudy weather. A 5.5-h sequence of good SOPHIE reference observations of HD 80606 could also be performed on the 2010 January 15 night. 24 and 33 exposures were secured during the two nights respectively. The exposure times ranged between 5 and 20 min, with typical values around 9 min; we tuned it in order to maintain a constant signal-to-noise ratio per pixel of $\sim 58$ at $550 \mathrm{~nm}$ despite the weather changes (seeing and absorption).

The measurements were performed with the same setup as the one we used for our observation of the 2009 February transit (Moutou et al. 2009). We used the fast-read-out-time mode of the CCD detector to minimize overheads. Observations were secured in high-resolution mode allowing the resolving power $\lambda / \Delta \lambda=75000$ to be reached. The first optical-fiber aperture was put on the target and the second on the sky; this allowed us to check that no diffuse light was polluting the HD 80606 spectra in these Moonless nights. Wavelength calibrations with a thorium lamp were performed with a $\sim 2$-h frequency each night, allowing the interpolation of the spectral drift of SOPHIE for the time of each exposure. A few exposures were performed with simultaneous thorium-lamp light in the second aperture to allow for simultaneous wavelength calibration; those extra-calibration did not significantly improve the radial velocity accuracy.

\subsection{Data reduction}

We used the SOPHIE pipeline (Bouchy et al. 2009) to extract the spectra from the detector images, to cross-correlate them with a G2-type numerical mask, then to fit the cross-correlation functions (CCFs) by Gaussians to get the radial velocities (Baranne et al. 1996; Pepe et al. 2002). Each spectrum produces a clear $\mathrm{CCF}$, with a $7.82 \pm 0.03 \mathrm{~km} \mathrm{~s}^{-1}$ full-width-at-half-maximum and a contrast representing $48.8 \pm 0.4 \%$ of the continuum. The accuracy of the measured radial velocities is typically around $4 \mathrm{~m} \mathrm{~s}^{-1}$. This includes photon noise (typically $\sim 2 \mathrm{~m} \mathrm{~s}^{-1}$ ), wavelength calibration $\left(\sim 2 \mathrm{~m} \mathrm{~s}^{-1}\right)$, and guiding errors $\left(\sim 3 \mathrm{~m} \mathrm{~s}^{-1}\right)$ that produce motions of the input image within the fiber (Boisse et al. 2010a). We also re-reduced the SOPHIE observations presented in Moutou et al. (2009) to have a uniform dataset reduced with the same version of the pipeline. There was no significant change by comparison with the data presented in Moutou et al. (2009), except for one of the reference exposures performed out of the transit, for which the correction due to the Moon pollution was significantly improved. SOPHIE measurements of HD 80606 performed in 2009 February and 2010 January have the same properties, except for the better signal-to-noise ratios for the shorter exposure times obtained in 2010, which is largely due to the primary mirror of the telescope that was realuminized in 2009 October.

The SOPHIE radial velocities of HD 80606 are plotted in Figs. 3 and 7 together with other data sets.

In the SOPHIE spectra, the cores of the large CaII H \& $\mathrm{K}$ absorption lines of HD 80606 at $3934.8 \AA$ and $3969.6 \AA$ show no chromospheric emissions. The level of the Ca II emission corresponds to $\log R_{\mathrm{HK}}^{\prime}=-5.3 \pm 0.1$ according to the SOPHIE calibration (Boisse et al. 2010b). For a G-type star $(B-V=0.76)$ with this level of activity, Santos et al. (2000) predict a dispersion below $5 \mathrm{~m} \mathrm{~s}^{-1}$ for the activity-induced stellar jitter. According 

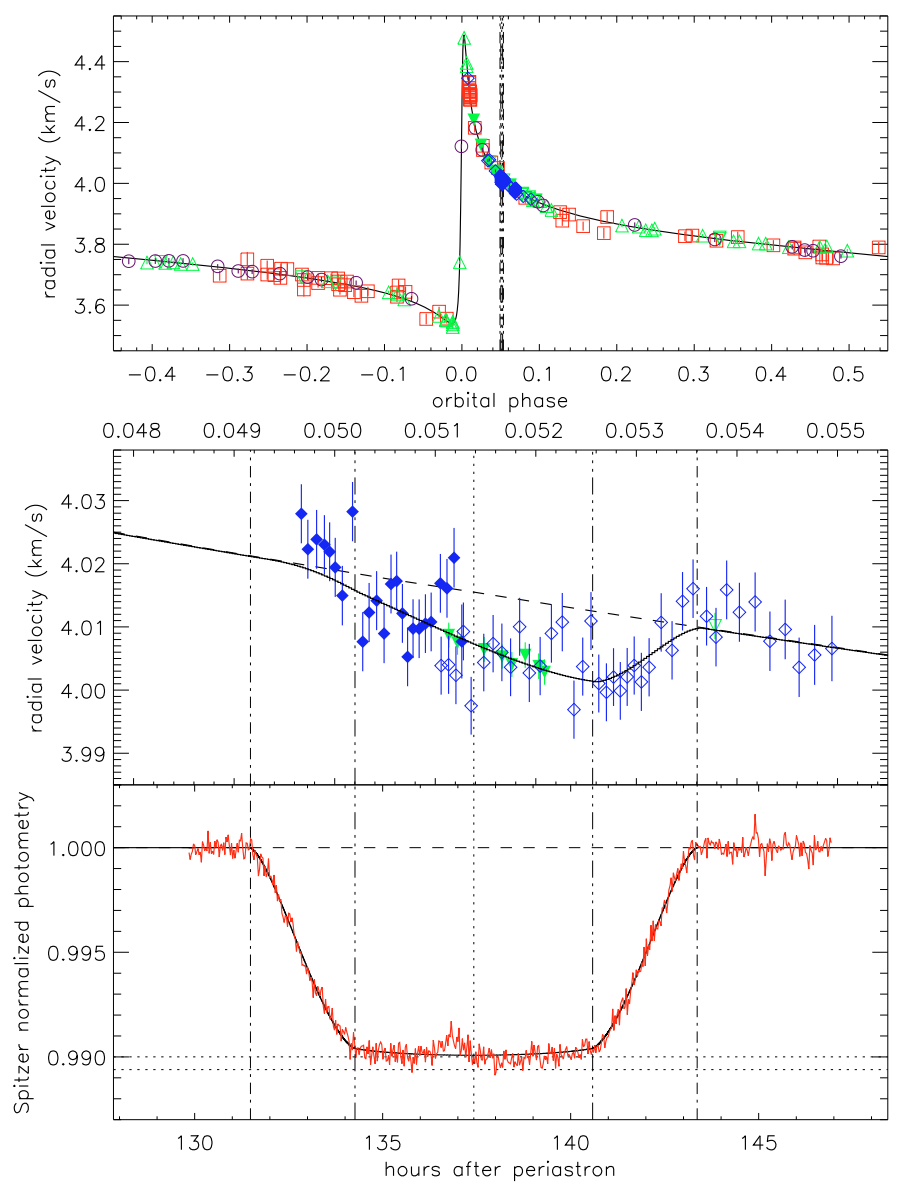

Fig. 3. Radial velocities and photometry of HD 80606 as a function of the orbital phase or the time after periastron. Upper panel: radial velocities as measured with ELODIE (red open squares, Moutou et al. 2009), HRS (purple open circles, Wittenmyer et al. 2009), HIRES (Winn et al. 2009a) pre- and post-upgrade (green open upward and downward triangles, respectively) and around the 2009 June transit (green filled downward triangles), and SOPHIE during the 2009 February and 2010 January transits (blue open and filled diamonds, respectively). Middle panel: same as above, but enlarged around the transit phase. Lower panel: Spitzer photometry during the 2010 January transit. The Spitzer data are binned per 250, i.e. one point each 100 s. The two horizontal dotted-lines show the absorption depth expected with the value $R_{\mathrm{p}} / R_{*}=0.100$ that we get and the value $R_{\mathrm{p}} / R_{*}=0.103$ measured in the visible by Pont et al. (2009) and Winn et al. (2009a). In all three panels the dashed lines show the models without transit and the solid lines show the models with transit (Rossiter-McLaughlin anomaly for radial velocities, and absorption feature for the light curve). The parameters of the fits are reported in Table 1. The vertical dotted lines show the mid-transit, the vertical dot-dashed lines show the first and fourth contacts, and the vertical dot-dot-dashed lines show the second and third contacts. The uncertainty on the timing of the mid-transit is $1.5 \mathrm{~min}$ (corresponding to $9 \times 10^{-6}$ in orbital phase), and about twice as much for the timing of the four contacts.

to Noyes et al. (1984) and Mamajek \& Hillenbrand (2008), this level of activity implies a stellar rotation period $P_{\text {rot }}>50$ days.

\section{Ground-based photometry}

In order to study its intrinsic variability, HD 80606 was also observed several nights before and after the 2010 January transit. Observations during the night of the transit were prevented by bad weather conditions in the observing site, located in Portalegre, Portugal. We gathered a total of nearly $23 \mathrm{~h}$ of observations in 10 nights spread from 2009 December 3 to 2010 January 25. The equipment used is a 12 -inch $f / 5.5$ MEADE LX200 telescope, and a SBIG ST8XME 9-micron-pixel CCD camera. The field is $28 \times 18.7 \mathrm{arcmin}^{2}$ and the pixel scale is $\sim 1.1 \mathrm{arcsec} /$ pixel. Observations were taken through a Bessell I filter, using integration times between 40 and $90 \mathrm{~s}$.

The frames were reduced with standard IRAF routines and aperture photometry was obtained for HD 80606, its companion HD 80607 and two additional reference stars located in the same field, located about 7 and 11.5 arcmin away from HD 80606. The size of the photometric aperture was varied on each night, depending on seeing conditions, to obtain the highest possible signal-to-noise ratio.

In every night, the flux ratio between HD 80606 and HD 80607 exhibits a smaller dispersion than the ratio of HD 80606 with any of the other two reference stars, or a combination of HD 80607 and the additional reference stars. Furthermore, the light curves obtained for HD 80607 - using the reference stars - also exhibit a larger dispersion than the flux ratio between HD 80606 and HD 80607. Therefore, the limits to the intrinsic variability of HD 80606 were set using only HD 80607 as reference star.

The root mean square for the different nights range from about 2.2 to $4.8 \mathrm{mmag}$, depending mainly on the weather conditions. During each night, no effect was seen due to variation of the sky level, or the position of the stars on the chip or the airmass. On the other hand, the nightly mean of our observations exhibits a dispersion of about $1.7 \mathrm{mmag}$ around its mean value.

Similar photometric observations of HD 80606 were conducted in the weeks around the 2010 January transit with the CCD camera at the $120-\mathrm{cm}$ telescope of the Haute-Provence Observatory with the setup used by Moutou et al. (2009). The filter used was $r_{\text {Gunn }}$ with a neutral density, and we did not defocus. Photometric observations started on January 6 and ended on January 23, with a total of eight sequences ranging from 30 to 120 min per night; in total, 187 images were acquired and analyzed. Exposure times of 10 to $120 \mathrm{~s}$ were used to account for varying transparency. An observation of the transit during the January-13 night could be performed with this instrument, on a coverage similar to this of the SOPHIE observations reported in Sect. 3. This ground-based photometry of a part of the transit will be studied in a forthcoming paper.

The flux was extracted from aperture photometry using the GCNTRD and APER procedures (Sect. 2.2) on a 8-pixel radius (0.69 $\mathrm{arcsec} / \mathrm{pixel})$. The background was estimated from a sky annulus with radii of 10 to 12 pixels. The root mean square of flux variations ranges from 1.0 to $3.1 \mathrm{mmag}$ per night, and the residual fluctuation has a standard deviation of $3.2 \mathrm{mmag}$ when the transit night is excluded. This is significantly larger than what was observed in Portalegre, which may indicate a real tendency of rotational modulation, or a significant level of systematics. No attempt was done to correct for the long-term behavior in the data, which appears to be compatible with the expected rotational period of the star (as discussed in Sect. 6.5).

We conclude therefore that HD 80606 is photometrically stable at the level of a few mmag in the optical range on the timescale of several weeks.

\section{Analysis}

We fitted this whole dataset to refine the system parameters. As a full transit was observed with Spitzer, possible systematic effects due to the combination of transit portions secured with different ground-based instruments are expected to be reduced here. 
In addition, together with the new radial velocities secured at phases previously uncovered, the constraints on the spin-orbit angle would be better.

\subsection{Combined fit}

\subsubsection{Method}

We first performed a combined fit of our Spitzer photometry of the 2010 January transit together with the available radial velocities of HD 80606. We also included in this combined fit the timing constraint on the eclipse as obtained from previous Spitzer measurements by Laughlin et al. (2009) and re-analyzed by Gillon (2009). As those data are not accurate enough to allow the ingress and egress of the eclipse to be significantly measured, we used the estimated epochs of the mid-time of these two events: HJD $=2454424.700 \pm 0.005$ and $2454424.775 \pm 0.005$.

We did not include in this combined fit the radial velocity measurements secured during and near the transits. Indeed, the Rossiter-McLaughlin observations do constrain the projected stellar rotational velocity $V \sin I_{*}$ and the sky-projected angle $\lambda$ between the planetary orbital axis and the stellar rotation axis, but they do not significantly constrain the parameters that we measured here in the combined fit. The analysis of the RossiterMcLaughlin data is presented below in Sect. 5.2 and takes into account the results of the combined fit presented here in Sect. 5.1. Thus, the combined fit uses the radial velocities secured with the instruments ELODIE at OHP (Moutou et al. 2009), HRS at HET (Wittenmyer et al. 2009), and HIRES at Keck (Winn et al. 2009a). This covers a 9.5-year span. The SOPHIE data are used only for the Rossiter-McLaughlin fit. We note that the ELODIE and SOPHIE radial velocities are absolute heliocentric, whereas those from HIRES and HRS are relative.

\subsubsection{Transit light curve of planets on eccentric orbit}

To calculate the transit light curve with a given set of orbital parameters (period, orbital inclination, semi major axis in unit of stellar radii, eccentricity and longitude of periastron), we calculated the sky projected distance between the planet's and the star's center in units of stellar radius; this last result was then directly put into the Mandel \& Agol (2002) algorithm with limb darkening coefficients. From a theoretical model (Kurucz 1979), the three non-linear limb-darkening coefficients at $4.5 \mu \mathrm{m}$ as defined by Sing (2010) were derived with $T_{\text {eff }}=5500 \mathrm{~K}$ and $\log g=4.5: c_{2}=0.89502981, c_{3}=-1.1230710$ and $c_{4}=0.46541027$. The limb darkening is low at this wavelength; Désert et al. (2009) showed that the uncertainties in the coefficients have no significant effects on the parameters derived from the transit light curve.

It is worth to note that the speed of light in the system has to be taken into account when comparing the times of the transit, the eclipse and the periastron. The transit takes place when the planet is at $\sim 0.29 \mathrm{AU}$ from the star (see Fig. 1); this implies an apparent advance of about $2.5 \mathrm{~min}$ for the transit. Similarly, the planet is at $\sim 0.03 \mathrm{AU}$ from the star at the eclipse, which implies a delay of $15 \mathrm{~s}$. Also because the planet-observer distance decreases during the transit, the speed of light correction makes the transit appears to last $8 \mathrm{~s}$ less than it really does. In the procedure we implemented this speed of light correction.

The orbit of HD 80606b is highly eccentric, and the transit takes place $\sim 5.7$ days after the periastron, when the planet is rapidly moving away from the star. During the transit, the starplanet distance increases by about $5.8 \%$ between the first and

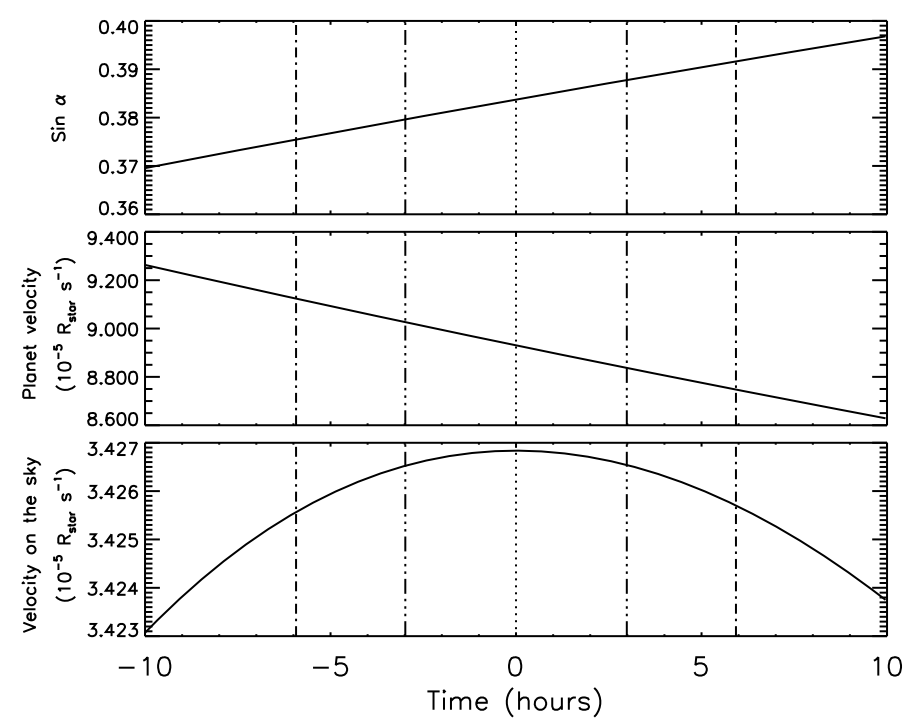

Fig. 4. Plot of the orbital velocity of the planet and orbit direction during the transit. $\alpha$ being the angle between the direction of the planet motion and the line of sight, $\sin \alpha$ is the fraction of the planet motion projected onto the sky (upper panel). Although the planet velocity significantly decreases during the transit (middle panel), the change of the orbital direction has the opposite effect such that the planet velocity on the sky is nearly constant (bottom panel). Even more, the planet velocity on the sky is nearly symmetrical around the center of the transit, reinforcing the apparent symmetry of the transit light curve. On the three panels, the vertical dotted lines show the mid-transit, the vertical dot-dashed lines show the first and fourth contacts, and the vertical dot-dot-dashed lines show the second and third contacts. Figure 1 shows these parameters drawn in a sketch.

the fourth contact, and the orbital velocity decreases by about $4.1 \%$ (Fig. 4). With that in mind, it would a priori suggest that the transit light curve could be highly asymmetrical with for instance an egress lasting longer than the ingress. However, during the transit, the direction of the planet motion also varies by about the same amount (upper panel of Fig. 4). If we define $\alpha$ as the angle between the direction of the planet motion and the line of sight, $\sin \alpha$ is the fraction of the planet motion projected onto the sky (see insert in Fig. 1). Between the first contact and the last contact, $\sin \alpha$ increases by about $4.3 \%$, exactly compensating for the decrease of the orbital velocity. As a result, the planet velocity on the sky is nearly constant. In addition to the low variation of the apparent planet velocity on the sky during the transit, it appears that the time variation of this velocity is nearly symmetrical around the center of the transit (bottom panel of Fig. 4). Consequently, the apparent velocity during the egress is extremely close to the velocity during the ingress, the latter being only $0.004 \%$ smaller than the former. This reinforces the apparent symmetry of the transit light curve. With the parameters of the best fit, the ingress duration (time between first and second contact) is predicted to last only half a second more than the egress duration (time between third and fourth contact), while both last about two hours and $45 \mathrm{~min}$.

This surprising symmetry for the transit light curve of a planet on a highly eccentric orbit can be explained by considering the projection on the sky of the gravitational force from the star to the planet. Because the transited star is the same as the attracting star, the component of the gravitational force projected on the sky is close to zero during the transit. As a consequence, the apparent velocity of the planet projected on the sky is nearly constant. Even more, the projected force is also symmetrical 
around the star center for various positions of the planet, which explains the symmetry of the time variation of the projected velocity around the center of the transit. As a conclusion, contrary to the common-sense idea, in any configuration the transit of a planet in front of its parent star is expected to be highly symmetric, even for extremely eccentric orbits (see also Winn 2010). Our Spitzer observations confirm this result for the highly eccentric HD 80606b's orbit.

\subsubsection{Free parameters}

Our combined fit of the radial velocities, the Spitzer transit light curve and the eclipse timing includes 19 free parameters. We list them below, classified in four categories depending on their nature and the dataset that constrain them:

1. two free parameters constrained by photometry only:

$-R_{\mathrm{p}} / R_{*}$, the ratio of the planetary and stellar radii;

- $a / R_{*}$, the semimajor axis in units of stellar radius;

2. six free parameters constrained both by radial velocities and photometry:

- $a \cos i / R_{*}$, the "standard" impact parameter, which is different (for an eccentric orbit) from the actual impact parameter $b=d_{\mathrm{t}} \cos i / R_{*}$, where $d_{\mathrm{t}}$ is the star-planet distance at mid-transit and $i$ is the inclination of the orbit;

- $P$, the orbital period of the planet, which is proportional to $\left(a / R_{*}\right)^{3}$, and to the inverse of the stellar density $\rho_{*}$ from the Kepler third law;

- $T_{0}$, the epoch of the periastron of the planet;

$-e \cos \omega$ and $e \sin \omega$, which constrain the two correlated parameters $e$ (the eccentricity of the planetary orbit) and $\omega$ (the longitude of its periastron);

- $K \sqrt{1-e^{2}}$, that depends both on the eccentricity $e$ and on the semi-amplitude $K$ of the radial velocity variations $K$ actually is constrained by radial velocities only, and is proportional to $M_{\mathrm{p}}$ and $\left(M_{*}\right)^{2 / 3}, M_{\mathrm{p}}$ and $M_{*}$ being the planetary and stellar masses;

3. four free parameters constrained by radial velocities only:

- $V_{n=1 \rightarrow 4}$, the center-of-mass radial velocities for each of the four radial velocity dataset used, namely ELODIE (Moutou et al. 2009), HRS (Wittenmyer et al. 2009) and HIRES whose pre- and post-upgrade data are considered as two different datasets (Winn et al. 2009a);

4. seven free parameters linked to the Spitzer light curve extraction (see Sect. 2.2):

- the two parameters $A_{j=1 \rightarrow 2}$ for the baseline;

- the five parameters $K_{i=1 \rightarrow 5}$ for the pixel-phase effect.

The direct, absolute measurements of $M_{*}$ and $R_{*}$, and consequently those of $M_{\mathrm{p}}$ and $R_{\mathrm{p}}$, are not feasible from this fit; none of those four parameters is measurable independently of the other ones from our dataset. One exception could be the stellar mass that could be directly determined through the semi-major axis $a$ thanks to the third Kepler law. Indeed, by measuring the delay of the eclipse mid-time and the advance of the transit's one that are due to the time the light takes to propagate through the HD 80606 system ( 2.5 min difference between those two events by comparison to the ephemeris, see Sect. 5.1.2), the semi-major axis could be theoretically directly measured in Astronomical Units. The uncertainties in the transit and eclipse measured mid-times are however on the order of 1.5 and 4 minutes respectively, which implies an accuracy on $a$ on the order of $0.5 \mathrm{AU}$ with this approach; this is not constraining here.
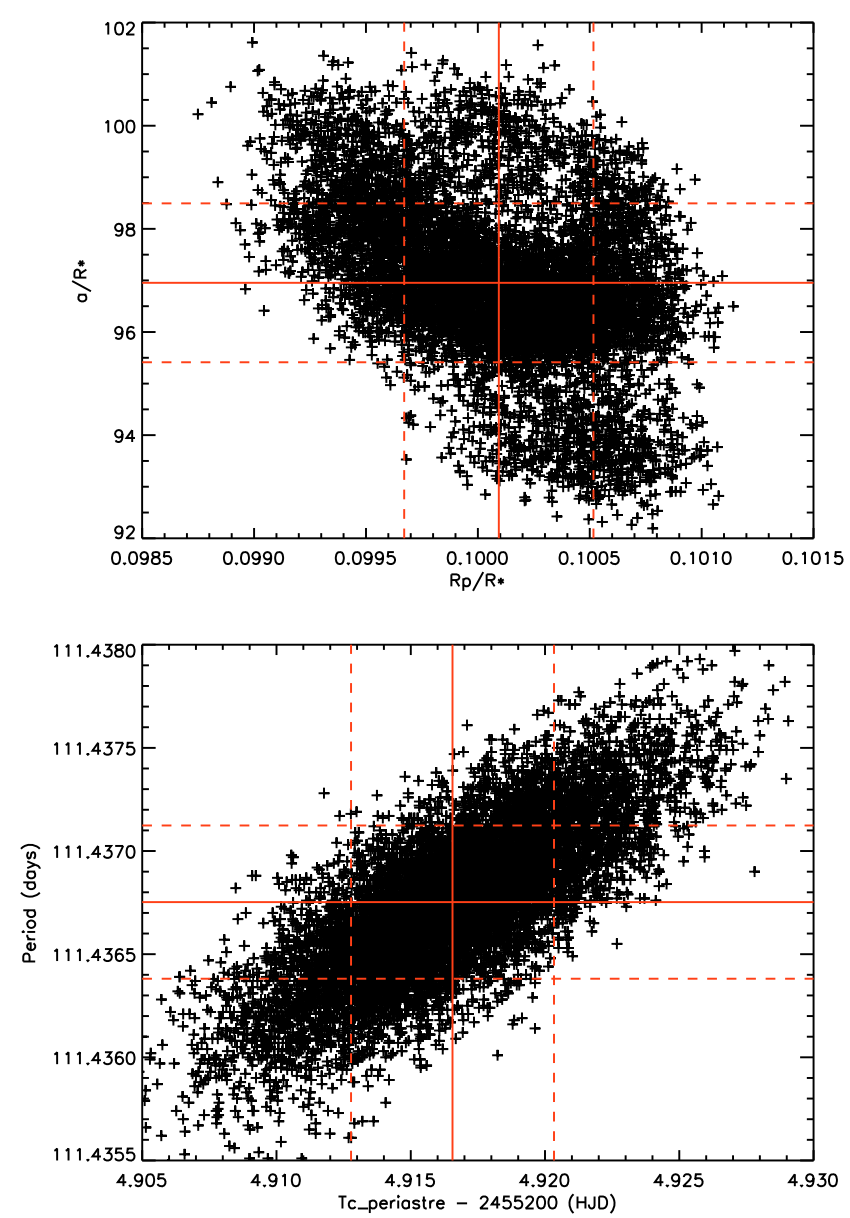

Fig. 5. Distributions of the parameters obtained for the 15000 fits performed with the prayer bead and bootstrap (Sect. 5.1.4). As an example, four parameters are plotted here: $a / R_{*}$ vs. $R_{\mathrm{p}} / R_{*}$ and $P$ vs. $T_{0}$ (periastron). The mean values and the standard deviations are shown.

Stellar evolution models remain thus mandatory to estimate $M_{*}$. Moutou et al. (2009) used isochrones to get $M_{*}=$ $0.98 \pm 0.07 M_{\odot}$ from $T_{\text {eff }}=5574 \pm 50 \mathrm{~K}, \log g=4.45 \pm 0.05$ and $[\mathrm{Fe} / \mathrm{H}]=0.43$ dex. This accuracy is typical for stellar evolution models, from which it is difficult to predict stellar masses at better than $\pm 10 \%$ (Fernandes \& Santos 2004). With additional constraints from the transit light curve, Pont et al. (2009) and Winn et al. (2009a) estimated the stellar mass of HD 80606 to $0.97 \pm 0.04$ and $1.05 \pm 0.032 M_{\odot}$, respectively. The $2-\sigma$ disagreement between the two estimates shows that here we probably reach the limit of the accuracy that is now achievable. Below we adopt the conservative interval $M_{*}=1.01 \pm 0.05 M_{\odot}$ that takes into account those two studies.

\subsubsection{Best parameters and error bars}

We used the Prayer Bead method (Moutou et al. 2004; Gillon et al. 2007) as applied by Désert et al. (2009) to compute the mean values of the free parameters and their statistic and systematic uncertainties (see examples in Fig. 5), together with the Levenberg-Marquardt algorithm to provide the best fit at each iteration of our procedure. This method was applied to the Spitzer photometry to account for possible correlated noise in the error budget. We simultaneously applied a bootstrap procedure to the radial velocity measurements, after having quadratically added a 
systematic uncertainty to the radial velocity datasets to put their corresponding reduced $\chi^{2}$ to unity. Thus we quadratically added $12 \mathrm{~m} \mathrm{~s}^{-1}$ to the ELODIE uncertainties, and 5.0 and $1.7 \mathrm{~m} \mathrm{~s}^{-1}$ to the HIRES uncertainties (pre- and post-upgrade, respectively). In total, 15000 shifts and fits of transit light curves and bootstraps of radial velocity errors were produced to derive the set of parameters and to extract their means and their corresponding standard deviations. In addition, we performed additional fits with the light curve considering data starting at ten different epochs before the ingress; by performing a prayer bead on 1500 shifts for each of those ten fits, we could estimate the errors caused by the uncertainty in the shape of the out-of-transit baseline (see Sect. 2.2).

The averaged values we obtained for the seven fitted parameters linked to the Spitzer light curve extraction (Sect. 2.2) are $A_{1}=58818$ and $A_{2}=6.860$ for the baseline, and $K_{1}=0.2142$, $K_{2}=0.1538, K_{3}=0.1083, K_{4}=0.0729$, and $K_{5}=0.0050$ for the pixel-phase effect. However, those parameters could be different at different epochs and for different pixel locations, so this is not clear whether the $A_{j}$ and $K_{i}$ values we derived here could be applied to other IRAC observations with Warm-Spitzer.

The best solution of the combined fit is plotted in Fig. 3, with a ramp cut up to $\mathrm{HJD}=-2455210.325$, i.e. the first $1.8 \mathrm{~h}$ of the observation were discarded, corresponding to the first 15000 over the 159232 unbined frames. The upper panel of Fig. 3 shows the radial velocities and the lower panel shows the Spitzer photometry binned per 250 pixels (the middle panel shows the Rossiter-McLaughlin fit that is discussed below in Sect. 5.2).

The derived parameters are reported in Table 1 together with their error bars; they are ranked as a function of the way they are derived. First are reported the free parameters of the combined fit that are listed in Sect. 5.1.3 and which are constrained by photometry only, then those that are constrained both by radial velocities and photometry, and then those constrained by radial velocities only. In this last category, the dispersions around the obtained radial velocity shifts are also reported. Twelve adjusted parameters of the combined fit are reported here. Then Table 1 shows the parameters that are directly derived from the above free parameters of the fit, without any additional hypothesis. This includes the transit and eclipse timings, the latest being obtained from Laughlin et al. (2009) and Gillon (2009). The following parameters are those that are derived by assuming the stellar mass $M_{*}=1.01 \pm 0.05 M_{\odot}($ Sect. 5.1.3) together with the parameters derived above and through the Kepler third law. The last parameters set in Table 1 are those relative to the Rossiter-McLaughlin fit that are obtained below in Sect. 5.2.

The dispersion of the Spitzer photometry around the transit light curve fit represents $5.3 \times 10^{-3}$ of the stellar flux for unbinned frames. This is the expected level of the photon noise. The amplitude of the correlated noise as seen for different binsizes of the light curve is on the order of $1.4 \times 10^{-4}$ of the stellar flux (Fig. 6). A bump in the light curve with an amplitude of $\sim 1$ mmag is seen just before the transit mid-time (see Sect.6.1). It could be instrumental or due to a spot (see Sect. 6.1). We performed fits without taking these points into account; this did not change significantly the derived parameters.

\subsection{Rossiter-McLaughlin fit}

The radial velocity measurements secured during transits were fitted to measure the sky-projected angle $\lambda$ between the planetary orbital axis and the stellar rotation axis. The data we used are the SOPHIE observations of the 2009 February transit
Table 1. Parameters for the HD 80606 system.

\begin{tabular}{|c|c|c|}
\hline Parameters & Values and 1- $\sigma$ error bars & Unit \\
\hline \multicolumn{3}{|c|}{ Free parameters constrained by photometry only } \\
\hline$R_{\mathrm{p}} / R_{*}$ & $0.1001 \pm 0.0006$ & \\
\hline$a / R_{*}$ & $97.0 \pm 1.6$ & \\
\hline \multirow{2}{*}{\multicolumn{3}{|c|}{ Free parameters constrained both by photometry and radial velocities }} \\
\hline & & \\
\hline$P$ & $111.4367 \pm 0.0004$ & days \\
\hline$T_{0}$ (periastron) & $2455204.916 \pm 0.004$ & HJD \\
\hline$e \cos \omega$ & $0.4774 \pm 0.0018$ & \\
\hline$e \sin \omega$ & $-0.8016 \pm 0.0017$ & \\
\hline$K \sqrt{1-e^{2}}$ & $171.1 \pm 0.5$ & $\mathrm{~m} \mathrm{~s}^{-1}$ \\
\hline \multicolumn{3}{|c|}{ Free parameters constrained by radial velocities only } \\
\hline$V_{\mathrm{ELODIE}}$ & $\begin{array}{c}3.7888 \pm 0.0023 \\
17.8\end{array}$ & $\begin{array}{c}\mathrm{km} \mathrm{s}^{-1} \\
\mathrm{~m} \mathrm{~s}^{-1}\end{array}$ \\
\hline$V_{\mathrm{HRS}} \underset{\sigma(\mathrm{O}-\mathrm{C})}{ }$ & $\begin{array}{c}-0.0193 \pm 0.0019 \\
6.3\end{array}$ & $\begin{array}{c}\mathrm{km} \mathrm{s}^{-1} \\
\mathrm{~m} \mathrm{~s}^{-1}\end{array}$ \\
\hline $\begin{array}{c}V_{\text {HIRES, pre-ugrade }} \\
\sigma(\mathrm{O}-\mathrm{C})\end{array}$ & $\begin{array}{c}-0.1845 \pm 0.0010 \\
5.5\end{array}$ & $\begin{array}{c}\mathrm{km} \mathrm{s}^{-1} \\
\mathrm{~m} \mathrm{~s}^{-1}\end{array}$ \\
\hline $\begin{array}{c}V_{\text {HIRES, post-ugrade }} \\
\sigma(\mathrm{O}-\mathrm{C})\end{array}$ & $\begin{array}{c}-0.1827 \pm 0.0007 \\
2.4\end{array}$ & $\begin{array}{c}\mathrm{km} \mathrm{s}^{-1} \\
\mathrm{~m} \mathrm{~s}^{-1}\end{array}$ \\
\hline \multicolumn{3}{|l|}{ Directly derived parameters } \\
\hline$b$ & $0.808 \pm 0.007$ & \\
\hline$i$ & $89.269 \pm 0.018$ & $\circ$ \\
\hline$T_{\mathrm{t}}$ (transit mid-time) & $2455210.6420 \pm 0.0010$ & HJD \\
\hline Transit duration $T_{1-4}$ & $11.88 \pm 0.09$ & $\mathrm{~h}$ \\
\hline Transit duration $T_{1-2}=T_{3-4}$ & $2.78 \pm 0.10$ & $\mathrm{~h}$ \\
\hline$e$ & $0.9330 \pm 0.0005$ & \\
\hline$\omega$ & $300.77 \pm 0.15$ & $\circ$ \\
\hline$K$ & $475.3 \pm 2.0$ & $\mathrm{~m} \mathrm{~s}^{-1}$ \\
\hline$\rho_{*}$ & $1.39 \pm 0.07$ & $\mathrm{~g} / \mathrm{cm}^{3}$ \\
\hline$T_{\mathrm{e}}$ (eclipse mid-time) & $2454424.736 \pm 0.003^{\ddagger}$ & HJD \\
\hline Eclipse duration $T_{1-4}$ & $1.85 \pm 0.14^{\ddagger}$ & $\mathrm{h}$ \\
\hline \multicolumn{3}{|c|}{ Derived parameters assuming a $M_{\star}$-value } \\
\hline$M_{\star}$ & $1.01 \pm 0.05^{\dagger}$ & $M_{\odot}$ \\
\hline$R_{\star}$ & $1.007 \pm 0.024$ & $R_{\odot}$ \\
\hline$M_{\mathrm{p}}$ & $4.08 \pm 0.14$ & $M_{\text {Jup }}$ \\
\hline$R_{\mathrm{p}}$ & $0.981 \pm 0.023$ & $R_{\text {Jup }}$ \\
\hline$\rho_{\mathrm{p}}$ & $5.4 \pm 0.4$ & $\mathrm{~g} / \mathrm{cm}^{3}$ \\
\hline$a$ & $0.455 \pm 0.008$ & $\mathrm{AU}$ \\
\hline \multicolumn{3}{|l|}{ Rossiter-McLaughlin effect } \\
\hline$V \sin I_{*}$ & $1.7 \pm 0.3$ & $\mathrm{~km} \mathrm{~s}^{-1}$ \\
\hline$\lambda$ & $42 \pm 8$ & $\circ$ \\
\hline $\begin{array}{c}V_{\mathrm{SOPHIE} \text {, transit February } 09} \\
\sigma(\mathrm{O}-\mathrm{C})\end{array}$ & $\begin{array}{c}3.9162 \pm 0.0013 \\
4.3\end{array}$ & $\begin{array}{c}\mathrm{km} \mathrm{s}^{-1} \\
\mathrm{~m} \mathrm{~s}^{-1}\end{array}$ \\
\hline $\begin{array}{l}V_{\text {HIRES, post-ugrade, transit June } 09} \\
\sigma(\mathrm{O}-\mathrm{C})\end{array}$ & $\begin{array}{c}-0.1795 \pm 0.0011 \\
0.7\end{array}$ & $\begin{array}{l}\mathrm{km} \mathrm{s}^{-1} \\
\mathrm{~m} \mathrm{~s}^{-1}\end{array}$ \\
\hline $\begin{array}{l}V_{\mathrm{SOPHIE}} \text {, transit January } 10 \\
\sigma(\mathrm{O}-\mathrm{C})\end{array}$ & $\begin{array}{c}3.9018 \pm 0.0013 \\
5.2\end{array}$ & $\begin{array}{c}\mathrm{km} \mathrm{s}^{-1} \\
\mathrm{~m} \mathrm{~s}^{-1}\end{array}$ \\
\hline
\end{tabular}

Notes. ${ }^{\dagger}$ Combined value from Pont et al. (2009) and Winn et al. (2009a); (†) from Laughlin et al. (2009).

(Moutou et al. 2009) and the new ones we secured in 2010 January (Sect. 3). We also used the HIRES data of the 2009 June transit (Winn et al. 2009a).

We first measured the radial velocity shift of each dataset by comparison to the center-of-mass radial velocity references computed in Sect. 5.1. Radial velocity measurements performed near to but off the transit are mandatory to constrain those shifts. A lack of these reference observations could prohibit an accurate measurement of the spin-orbit angle. For example for the recent Rossiter-McLaughlin observation of the planetary system Kepler-8 (Jenkins et al. 2010), 


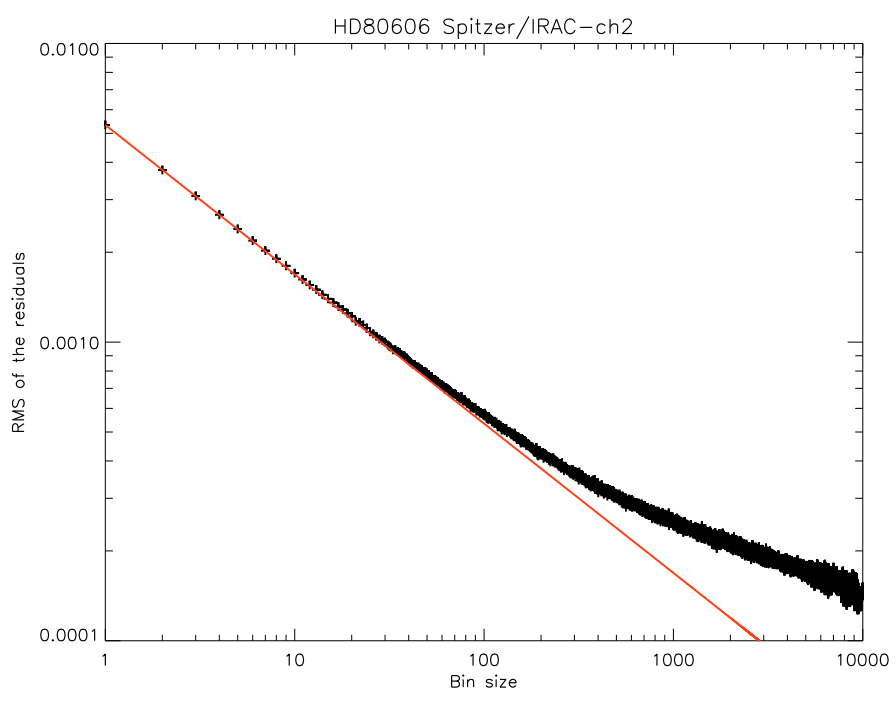

Fig. 6. Root mean square (RMS) of binned residuals of the Spitzer transit light curve as a function of the bin size $n$. The solid red line is proportional to $n^{-1 / 2}$, as expected if only photon noise is considered.

the paucity of off-transit observations makes it difficult to conclude if the apparent asymmetry of the Rossiter-McLaughlin shape by comparison to the Keplerian curve is due to an actual spin-orbit misalignment or to a shift due to another cause, as stellar activity and/or instrumental drifts.

For the 2009 February data of HD 80606, we used as reference the nine SOPHIE measurements performed the nights before and after the transit night, as well as those nine performed after the fourth contact the night of the transit. For the 2010 SOPHIE data we used the 33 exposures obtained the January-15 night, i.e. two days after the transit. Finally, for the HIRES data we used the six 2009 June measurements secured before and after the night of the transit (the 2009 June data were excluded from the HIRES post-upgrade dataset used in Sect. 5.1 for the fit of the orbit). The radial velocity shifts for the three datasets are reported in Table 1. We found a shift of $14.4 \pm 1.9 \mathrm{~m} \mathrm{~s}^{-1}$ between the 2009 and 2010 SOPHIE datasets, and a shift of $3.2 \pm 1.3 \mathrm{~m} \mathrm{~s}^{-1}$ between the HIRES measured in 2009 June and the other postupgrade HIRES data secured more than one year earlier. These shifts could result from a combination of instrumental effects, an unknown component in the system and/or activity-induced jitter.

The reference observations secured near each of the three transits are plotted in the right panels of Fig. 7. Using these offtransit radial velocities, we also computed the uncertainties we had to add to the radial velocities tabulated error bars in order to put to unity the reduced $\chi^{2}$ corresponding to the Keplerian fit. We thus quadratically added $1.0 \mathrm{~m} \mathrm{~s}^{-1}$ and $2.5 \mathrm{~m} \mathrm{~s}^{-1}$ to the SOPHIE measurements secured in 2009 and 2010 respectively, and $1.7 \mathrm{~m} \mathrm{~s}^{-1}$ to the HIRES measurements (in agreement with the other HIRES post-upgrade data used above, see Sect. 5.1.4).

We modeled the Rossiter-McLaughlin anomaly shape with the analytical approach developed by Ohta et al. (2005). The complete model has 14 parameters: the stellar limb-darkening linear coefficient $\epsilon$, the transit parameters $R_{\mathrm{p}} / R_{*}, a / R_{*}$ and $i$, the orbital parameters $\left(P, T_{0}, e, \omega, K\right)$, the three radial velocity shifts measured above, and finally $V \sin I_{*}$ and $\lambda$. We computed $\epsilon=0.722$ in the wavelength range $5300-6300 \AA$ with the same method as in Sect. 5.1.2. The transit and orbital parameters are fixed from the results obtained in Sect. 5.1; their uncertainties are
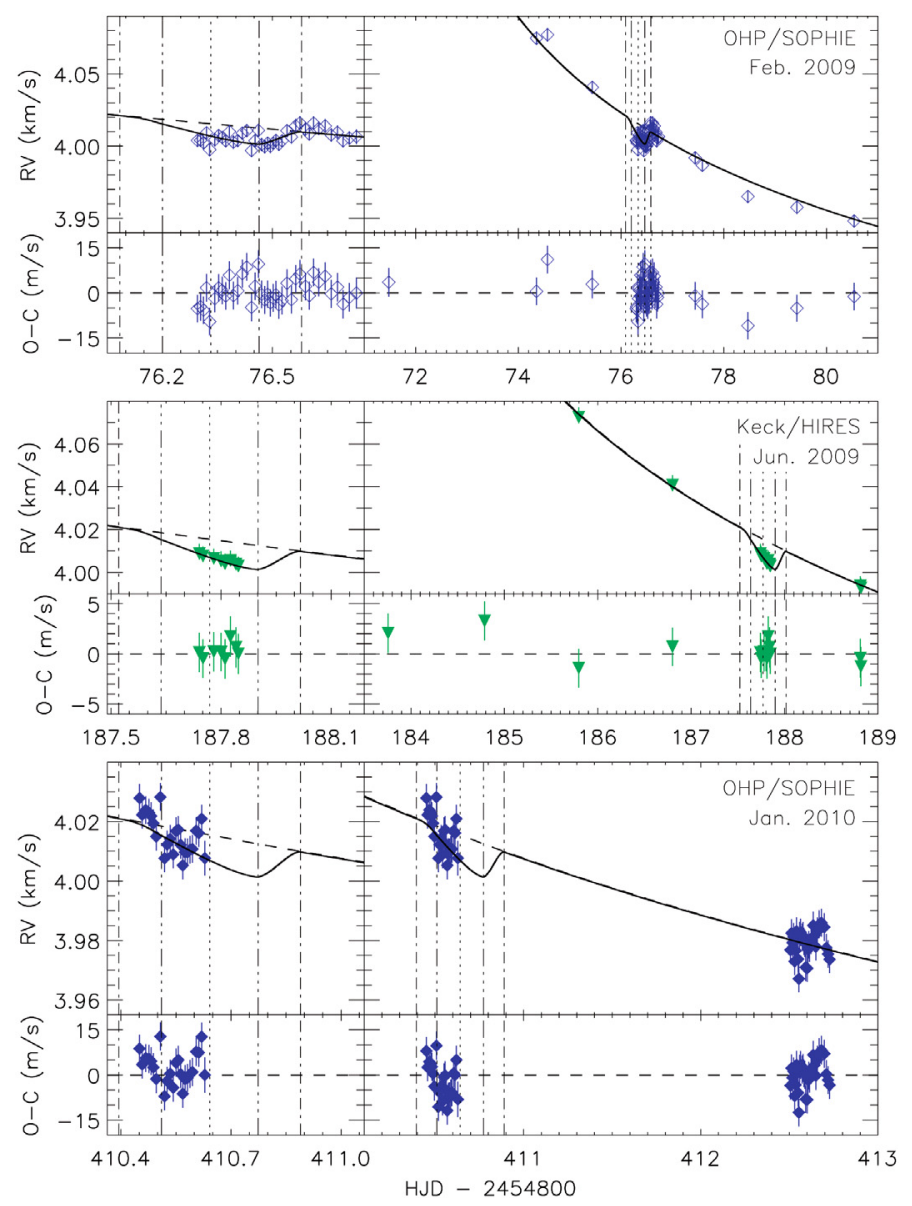

Fig. 7. Radial velocities of HD 80606b around transits and their Rossiter-McLaughlin fit. The upper panel shows the SOPHIE observations around the 2009 February 14 transit (Moutou et al. 2009), the middle panel shows the HIRES observations around the 2009 June 5 transit (Winn et al. 2009a), and the lower panel shows the SOPHIE observations around the 2010 January 13-14 transit (Sect. 3). On each of these three panels the data are plotted together with the $1-\sigma$ error bars. The fits with and without transit are the solid and dashed lines, respectively. The left panels show the data during the nights of the transits, and the right panels show all the data secured the nights before and after the transits to allow the measurement of the radial velocity shift of each dataset. The $\mathrm{O}-\mathrm{C}$ residuals of the fit including the Rossiter-McLaughlin anomaly are also plotted. On all panels the vertical dotted lines show the mid-transit, the vertical dot-dashed lines show the first and fourth contacts, and the vertical dot-dot-dashed lines show the second and third contacts.

negligible by comparison to those of the two main free parameters of the Rossiter-McLaughlin fit: $\lambda$, which is constrained by the asymmetry of the anomaly, and $V \sin I_{*}$, which is constrained by its amplitude.

The best fit is plotted in the middle panel of Fig. 3 and in Fig. 7. It is obtained for $\lambda=42^{\circ}$ and $V \sin I_{*}=1.7 \mathrm{~km} \mathrm{~s}^{-1}$; its $\chi^{2}$ is 117.8 for 117 degrees of freedom. The confidence interval contours estimated from $\chi^{2}$ variations (Hébrard et al. 2002) for the two correlated $\lambda$ and $V \sin I_{*}$ parameters are plotted in Fig. 8. The uncertainties obtained this way are $\pm 6^{\circ}$ and $\pm 0.2 \mathrm{~km} \mathrm{~s}^{-1}$, respectively. We increased them to take the uncertainties in the radial velocity shifts of the three datasets with respect to the Keplerian curve computed in Sect. 5.1 into account. Our final values are $\lambda=42^{\circ} \pm 8^{\circ}$ and $V \sin I_{*}=1.7 \pm 0.3 \mathrm{~km} \mathrm{~s}^{-1}$. We checked that the uncertainties of the parameters derived in Sect. 5.1 and used here in the Rossiter-McLaughlin fit imply 


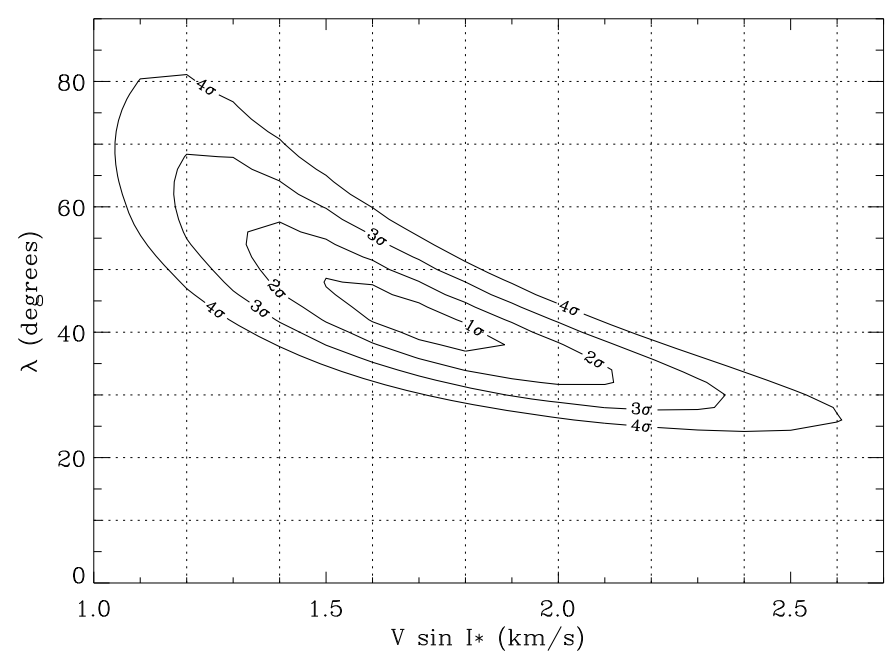

Fig. 8. Confidence interval contours for the $\lambda$ and $V \sin I_{*}$ values from the Rossiter-McLaughlin fit.

negligible uncertainties on $\lambda$ and $V \sin I_{*}$; similarly, the uncertainty in the $\epsilon$ limb-darkening coefficient is negligible.

The adopted values are reported in Table 1, together with the dispersion of the radial velocities performed during the transit with respect to the Rossiter-McLaughlin fit. The dispersion is in the range $4-5 \mathrm{~m} \mathrm{~s}^{-1}$ for SOPHIE data; we have a similar dispersion around the Keplerian curve for the SOPHIE reference measurements performed before and after the transit. For the HIRES data, the dispersion around the Rossiter-McLaughlin fit is $0.8 \mathrm{~m} \mathrm{~s}^{-1}$, whereas it is twice as large $\left(1.7 \mathrm{~m} \mathrm{~s}^{-1}\right)$ for the 2009 June HIRES measurements performed before and after the transit. So it could remain a slight systematic in the HIRES radial velocities secured during the transit. Yet this effect is small and is detected here due to the particularly high accuracy of these measurements.

\section{Discussion}

\subsection{Warm-Spitzer transit light curve}

This planetary transit observation is among the first secured with Spitzer in its post-cryogenic mission. This shows that accurate transit light curves can be obtained in this second part of the observatory mission despite enhanced ramp and pixel-phase effects. Our Warm-Spitzer transit light curve of HD 80606 has lower uncertainties than those obtained from the ground. The typical durations of the pixel-phase effect $(70 \mathrm{~min})$ and the planetary transit $(12 \mathrm{~h})$ are on time-scales different enough to avoid significant uncertainties of the derived system parameters due to this instrumental effect. The transit light curve is well detected, without extra signatures of transiting rings or satellites. According to our accuracy, the signature of an hypothetic satellite would have been detected up to a magnitude depth slightly below $1 \mathrm{mmag}$; this corresponds to an upper limit of $2 R_{\oplus}$ on the radius.

A bump in the light curve is seen just before the transit midtime with an amplitude of $\sim 1 \mathrm{mmag}$. It could correspond to the planet occulting a dark spot during the transit (Pont et al. 2007; Rabus et al. 2009). The source HD 80606 is not an active star, so the probability of such an event is low. The stability at the level of a few mmag of the stellar flux observed in the optical (Sect. 4) would correspond to a stability below 1 mmag at
$4.5 \mu \mathrm{m}$, assuming dark spots are $1000-\mathrm{K}$ cooler than the stellar surface (Désert et al. 2010); so the optical photometry cannot exclude that the feature detected in the Spitzer light curve is due to a spot. One can note that at the time of this feature (near $137 \mathrm{~h}$ after the periastron in Fig. 3), a simultaneous feature is also seen in the SOPHIE radial velocity data. This could argue in favor of the interpretation of this event in terms of phenomenon at the surface of HD 80606. This seems unlikely though, because a photometric feature with this low flux amplitude should a priori not produce this high radial velocity effect. The radial velocity feature is more likely an instrumental systematic. The feature in the Spitzer light curve is detected at a maximal value of the pixel-phase effect (Fig. 2) so we should remain cautious about instrumental effects. We monitored the coordinates of the target on the subarray through the observation to test if the bump corresponds to a particular area of the detector. This is not the case: at the epoch of the bump, the target is located on a position of the detector where the target passes numerous times before and after this event, and where the pixel-phase effect apparently is well corrected by our procedure. So we found no strong reasons to particularly favor an instrumental effect to explain the presence of this feature in the Spitzer light curve. The most likely explanation is the small star spot on the surface HD 80606, above which the planet is transiting.

Our dataset results from the first joined campaign during which space-based photometry and high-precision radial velocities were carried out simultaneously. If large stellar spots are detected during a similar campaign, this would help to better understand the effect of stellar activity on radial velocity measurements. Stellar jitter indeed introduces noise in measured radial velocities and is a significant limitation in high-precision velocimetry (see e.g., Saar \& Donahue 1997; Santos et al. 2000; Boisse et al. 2009; Queloz et al. 2009; Hébrard et al. 2009a). No stellar spots large enough to be spectroscopically detected were apparently seen here.

\subsection{Comparison with previous measurements}

The HD 80606's system parameters that we report in Table 1 have a better accuracy than previous studies (Moutou et al. 2009; Pont et al. 2009; Gillon 2009; Winn et al. 2009a; Hidas et al. 2010). With respect to the ground observation of nearly all the transit phases used by Winn et al. (2009a), the uncertainties presented here are better by factors two to five. Exceptions are the parameters $e, \omega$ and $K$ for which the error bars are not significantly reduced. This is because most of the constraints on these three parameters come from the radial velocity on a 9.5-year time span already used by previous studies.

For most of the parameters, the revised, more accurate values agree within $1 \sigma$ with those previously published. There is a $2-\sigma$ disagreement on the inclination $i$ of the orbit, which is found in our study to be slightly lower; this implies a more grazing transit, a larger impact parameter $b$, and a slightly longer duration for ingress and egress ( $\sim 10 \mathrm{~min}$ longer). The $a / R_{*}$-ratio is also found to be $5 \%$ smaller, a shift by $2 \sigma$ according the error bars from Winn et al. (2009a).

The projected stellar rotational velocity we found, $V \sin I_{*}=$ $1.7 \pm 0.3 \mathrm{~km} \mathrm{~s}^{-1}$, is slightly higher than the value obtained by Winn et al. (2009a) from the Rossiter-McLaughlin fit of their HIRES data: $V \sin I_{*}=1.12_{-0.22}^{+0.44} \mathrm{~km} \mathrm{~s}^{-1}$. Whereas we also used their HIRES in our analysis, our different result is explained because we used additional SOPHIE data and measured a smaller $R_{\mathrm{p}} / R_{*}$ ratio (see Sect. 6.3). In addition, a difference with the 
study by Winn et al. (2009a) is the shift of $3.2 \pm 1.3 \mathrm{~m} \mathrm{~s}^{-1}$ that we found between the HIRES data secured near the transit and the other ones (Sect. 5.2); this implies a slightly larger amplitude for the Rossiter-McLaughlin anomaly, thus a larger $V \sin I_{*}$. The value $V \sin I_{*}=1.7 \pm 0.3 \mathrm{~km} \mathrm{~s}^{-1}$ that we measured nonetheless agrees with those found from synthetic spectral fitting: $V \sin I_{*}=1.8 \pm 0.5$ and $2.0 \pm 0.5 \mathrm{~km} \mathrm{~s}^{-1}$ by Valenti \& Fischer (2005) and Winn et al. (2009a), respectively. The discrepancy noted and discussed by several authors (Winn et al. 2005; Triaud et al. 2009; Hirano et al. 2010; Simpson et al. 2010) between the $V \sin I_{*}$ measured from the Rossiter-McLaughlin effect and from the spectral modeling of line broadening is negligible for such a slow-rotating star like HD 80606.

The stellar radius we measured, $R_{\star}=1.007 \pm 0.024 R_{\odot}$, is slightly larger than that obtained by Winn et al. (2009a), namely $R_{\star}=0.968 \pm 0.028 R_{\odot}$. Both values remain in agreement with the one deduced by Moutou et al. (2009) from relationships between stellar radius, luminosity, temperature, gravity and mass, namely $R_{*}=0.98 \pm 0.07 R_{\odot}$.

The radius ratio $R_{\mathrm{p}} / R_{*}$ and the timing of the transit that we found significantly differ from the values derived from groundbased observation; this is discussed below.

\subsection{Planetary radius}

The radius ratio we found from the transit observed with Spitzer is $R_{\mathrm{p}} / R_{*}=0.1001 \pm 0.0006$. By comparison, Winn et al. (2009a) measured $R_{\mathrm{p}} / R_{*}=0.1033 \pm 0.0011$; Pont et al. (2009) found the same radius ratio as Winn et al. (2009a), but with an uncertainty three time larger, mainly due to the lack of ingress observation. The radius ratio measured in the infrared from space is thus $\sim 3 \%$ smaller than the one measured in the optical from ground. This is a $3-\sigma$ difference according the error bar reported by Winn et al. (2009a). The two horizontal dotted lines in the lower panel of Fig. 3 show the absorption depths expected with the two values; the infrared radius ratio clearly is smaller than the optical one.

The uncertainties in the background measurement (Sect. 2.2) are too small to account for such a radius difference. A variation of the stellar brightness due to spots could explain timevariations in the measured radius ratio (Désert et al. 2010). The upper limit of the brightness variations of the non-active star HD 80606 (Sect. 4) is however too small to explain a $2 \%$ radius-ratio variation. The planetary thermal emission could produce an underestimation of the measured radius ratio (Kipping \& Tinetti 2010). The emission level measured by Laughlin et al. (2009) at $8 \mu \mathrm{m}$ near the periastron is higher than the planetary emission expected at $4.5 \mu \mathrm{m}$ at the transit; even with this overestimated planetary flux, the effect would be negligible according the error bars on the radius ratio we measure at $4.5 \mu \mathrm{m}$.

The question of possible interpretation in terms of differential atmospheric absorption could be raised. The planet apparent radius as a function of the wavelength has been shown to follow the equation $\mathrm{d} R_{\mathrm{p}} / \mathrm{d} \lambda=H \mathrm{~d} \ln \sigma / \mathrm{d} \lambda$ (Lecavelier des Etangs et al. $2008 \mathrm{a}, \mathrm{b})$, where $\lambda$ is the wavelength, $\sigma$ is the cross section of the mean atmospheric absorber, $H=k T / \mu g$ is the atmospheric scale height, $k$ is the Boltzmann constant, $T$ is the temperature, $\mu$ is the molecular mass, and $g$ is the planet gravity which is about $100 \mathrm{~m} \mathrm{~s}^{-2}$. If the variation of the radius is due to the variation of the absorption by haze in the atmosphere as for the HD $189733 \mathrm{~b}$ (Pont et al. 2008), even assuming Rayleigh scattering which produces the steepest variation of absorption as a function of wavelength, a temperature of about $5000 \mathrm{~K}$ is required to interpret the present measurements, which is much higher than actually measured (Laughlin et al. 2009). Indeed, because of the large planetary mass the scale height is only $60 \mathrm{~km}$ at a typical temperature of $1000 \mathrm{~K}$. The difference of the measured radius is about 40 times this scale height and is therefore unlikely due to atmospheric differential absorption.

We found no astrophysical interpretations able to explain the radius difference between optical and infrared wavelengths. It is more likely that the error bars are slightly underestimated, maybe in the ground-based composite light curve. We note that since we obtain a larger stellar radius than Winn et al. (2009a), we obtain a similar planetary radius despite the different radius ratio: $R_{\mathrm{p}}=0.981 \pm 0.023 R_{\mathrm{Jup}}$.

\subsection{Transit timing}

The mid-times of the transit and the eclipse given in Table 1 are those that are measured. This means that the transit midtime $T_{\mathrm{t}}$ we report is in significant advance by about $2.5 \mathrm{~min}$ in comparison to that predicted from the epoch $T_{0}$ we report for the periastron of the planet (see Sect. 5.1.2). Indeed, this latest time is in the referential frame of the radial velocities, which are those of the star. Similarly, the epoch $T_{\mathrm{e}}$ of the eclipse reported in Table 1 is delayed by about $15 \mathrm{~s}$ compared with the ephemeris computed from $T_{0}$.

The mid-time of the transit we obtain is accurate at the level of $1.5 \mathrm{~min}$, whereas the accuracy is $6 \mathrm{~min}$ for the epoch of the periastron. Laughlin et al. (2009) obtained a 4-min accuracy for the mid-time of the eclipse. These accuracies are high, especially when they are compared with the long duration of the transit and the long orbital period. Using radial velocities alone, even if they span a time as long as 9.5 years, the error bars on the eclipse and periastron timing are three times larger. For the transit timing, the radial velocities cannot predict it at better than two to three hours. Transit and eclipse detections allow here more accurate timings.

This high-accuracy offers an opportunity to look for possible transit timing variations (TTVs). According to the mid-transit time and the period we found, we obtain for the 2009 February and June transits a mid-time that is $\sim 23$ min earlier than the $T_{\mathrm{t}}$ time measured by Winn et al. (2009a) from these events. Their accuracy on this timing was $\pm 7 \mathrm{~min}$, so the disagreement is at the level of $3 \sigma$. If it is not caused by underestimated systematic uncertainties, this difference in the transit timing could in principle be due to additional bodies in the system, like satellites of the transiting planet or additional planetary-mass bodies in the system (see e.g., Holman \& Murray 2005; Agol et al. 2005; Nesvorny \& Beaugé 2010).

We explored a small region of the parameter space of a hypothetical additional planet in search for a few examples that could explain a $\sim 20$-min difference between two nearby transits. To do this, we performed a series of 3-body simulations by integrating the equations of motion using the Burlisch-Stoer algorithm implemented in the Mercury6 package (Chambers 1999).

Planets in resonant orbits could explain such TTV even with masses small enough to prohibit their detection with the available radial velocities. For example, a 15-Earth-mass planet in a circular 4:1-resonant orbit produces the adequate timing anomalies, with many pairs of transits exhibiting a $\sim 20$-min discrepancy. The simulated timing variations exhibit an amplitude of $80 \mathrm{~min}$ in this case. A 0.17 -Jupiter-mass planet at the $6: 1$ resonance produces similar results, although the overall amplitude is reduced to $\sim 40 \mathrm{~min}$. Both cases would imply radial velocity 
variations with semi-amplitudes $K \simeq 1.5$ and $4 \mathrm{~m} \mathrm{~s}^{-1}$, respectively, so we cannot exclude those two cases: the first one would be undetectable in the available radial velocity dataset, whereas the second one would be at the limit of detection. More massive planets on non-resonant orbits could be excluded. For example, a planet located in a circular orbit at $3 \mathrm{AU}$ and having a mass of 1.7 Jupiter masses would imply a small number of pairs of nearby transits exhibit a variation of the order of $20 \mathrm{~min}$. It would imply radial velocity variations of $K \simeq 30 \mathrm{~m} \mathrm{~s}^{-1}$ which is detectable with the available radial velocities. In all these cases, the short-term variations (in timescales of the order of 2000 days) exhibited by the orbital parameters of the transiting planet remain below the level of precision reached from our combined fit (Sect. 5.1). However, the variations in timescales on the order of $10^{5-6}$ days are substantially larger in all cases. In particular, some 4:1-resonant cases could be unstable after a few thousands of years.

We also explored the possibility that the observed discrepancy was produced by a satellite of the transiting planet. In the more favorable case of a satellite orbiting the planet at the Hill's radius, we found that its mass must be around 3/100 that of HD $80606 \mathrm{~b}$ for it to be the cause of a 20 -min delay in the transit occurrence, which corresponds to 40 Earth masses.

\subsection{Spin-orbit misalignment}

We confirm the spin-orbit misalignment in the HD 80606 system and reduce the uncertainty in its measurement: $\lambda=42 \pm 8^{\circ}$. This allows scenarios with aligned spin-orbit to be rejected with a high level of confidence. This misalignment would imply an asymmetry in the Rossiter-McLaughlin anomaly as the star is not a perfect sphere but is slightly elongated at the equator due to its rotation. However, the accuracy of the data is not high enough to allow such a tiny effect to be detected.

The $\lambda$ angle measures the sky-projected angle between the planetary orbital axis and the stellar rotation axis. Its actual value remains unknown, as the inclination $I_{*}$ of the stellar rotation axis is undetermined. In cases where $\lambda=0$ is measured, it is reasonable to assume $I_{*} \simeq 90^{\circ}$ and an actual spin-orbit alignment. For cases like HD 80606, where $\lambda$ is significantly different from 0 , there is certainly no reason to assume the stellar rotation axis is parallel to the sky plan.

The projected stellar rotational velocity we get from the Rossiter-McLaughlin fit is $V \sin I_{*}=1.7 \pm 0.3 \mathrm{~km} \mathrm{~s}^{-1}$. According to our measured radius for HD 80606, $R_{\star}=1.007 \pm 0.024 R_{\odot}$, and to the relation from Bouchy et al. (2005), this $V \sin I_{*}$ translates into a stellar rotation period $P_{\text {rot }} / \sin I_{*}=30 \pm 5$ days. So the stellar rotation period apparently is shorter than 40 days. This is shorter than the limit $P_{\text {rot }}>50$ days we get in Sect. 3.2 from the low activity of HD 80606. This estimation based on the activity is not accurate especially for long rotation periods, though. These two values suggest a rotation period in the range of 40 50 days, so an $I_{*}$-angle near $90^{\circ}$. The value $\lambda=42 \pm 8^{\circ}$ that we measure is thus probably close to the value of the misalignment angle without projection effect. This means that the actual angle between the spin-axis of HD 80606 and the normal to the planetary orbital plane is about $40^{\circ}$.

The first case of a planetary system with a stellar spin misaligned with the normal of the planetary orbit was discovered by Hébrard et al. (2008) in the XO-3 system. This result was hereafter confirmed by Winn et al. (2009b), who however found a lower $\lambda$-value. The HD 80606-system was the second one reported to have a spin-orbit misalignement by

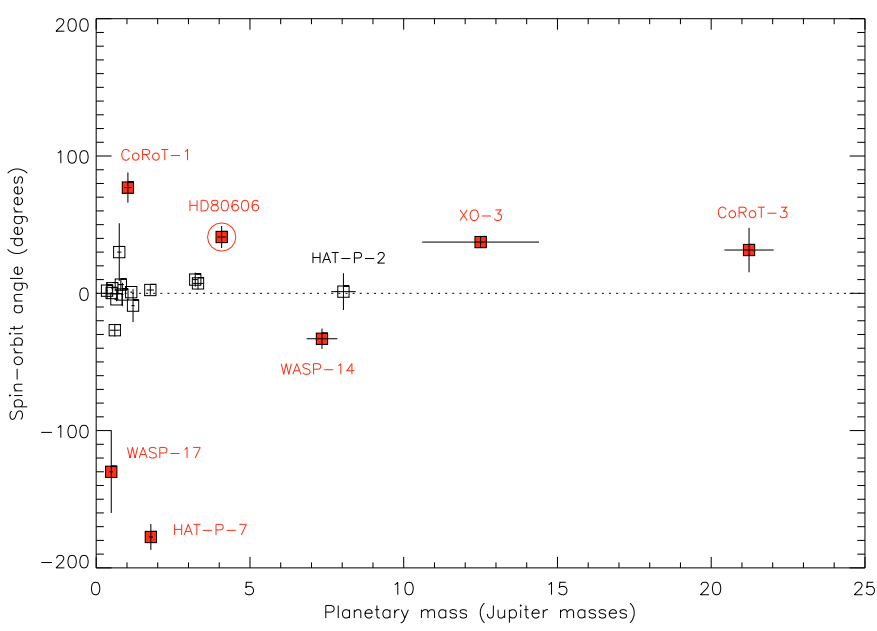

Fig. 9. Sky-projected $\lambda$-angle between the planetary orbital axis and the stellar rotation axis as a function of the planetary mass for 21 published systems (see references in Sect. 6.5). Systems with $|\lambda|>30^{\circ}$ are infilled, red symbols. The HD 80606-system is marked by a circle.

Moutou et al. (2009). This result was subsequently confirmed and refined successively by Pont et al. (2009), Gillon (2009), Winn et al. (2009a) and finally by the present study. Other planetary systems with significantly misaligned spin-orbit have been reported since then: WASP-14 (Johnson et al. 2009), WASP17 (Anderson et al. 2010), HAT-P-7 (Winn et al. 2009c; Narita et al. 2009a), and CoRoT-1 (Pont et al. 2010). There are thus now six known misaligned systems. Two other systems may be misaligned, but the large uncertainties in the reported $\lambda$-values prohibit definitive conclusion: TrES-1 (Narita et al. 2007) and CoRoT-3 (Triaud et al. 2009). The recent case of Kepler-8 (Jenkins et al. 2010) is presented as moderately misaligned, but this requires confirmation (see Sect. 5.2). On the other hand, twelwe systems are apparently aligned, namely HD 209458 (Queloz et al. 2000; Wittenmyer et al. 2005), HD 189733 (Winn et al. 2006; Triaud et al. 2009; Collier Cameron et al. 2010), HD 149026 (Wolf et al. 2007; Winn \& Johnson, in prep.), HD 17156 (Cochran et al. 2008; Barbieri et al. 2009; Narita et al. 2009b), HAT-P-1 (Johnson 2008), HAT-P-2 (Winn et al. 2007; Loeillet et al. 2008), HAT-P-13 (Winn et al. 2010), WASP-3 (Simpson et al. 2010; Tripathi et al. 2010), WASP-6 (Gillon et al. 2009), TrES-2 (Winn et al. 2008), TrES-4 (Narita et al. 2010), and CoRoT-2 (Bouchy et al. 2008).

Fabrycky \& Winn (2009) have shown that the whole sample of the measured $\lambda$-angles could be well reproduced with a bimodal distribution by assuming that a fraction of the orbits have random orientations relative to the stars and the remaining ones are perfectly aligned. The systems of misaligned orbits would be those which experienced gravitational interactions between planets and/or stars, like as Kozai migration.

In Fig. 9 we show the measured $\lambda$-angles as a function of the planetary masses for the 21 systems with published measurements reported above. This plot suggests a scenario with three distinct populations. Indeed, for the planets with masses similar to that of Jupiter, most of the spins are aligned with the orbits; this is expected for planets that formed in a protoplanetary disk far from the star and that slowly migrated closer-in at a later time. A small fraction of these Jupiter-mass planets however exhibits large $\lambda$-angles; this could be the signature of the second population in the scenario by Fabrycky \& Winn (2009), those which experienced gravitational interactions and that apparently 
are less frequent. These planets also seem to be those than can have extreme $\lambda$-values.

A third population could be formed by the large-mass planets. Indeed, most of them exhibit a misaligned spin-orbit (see also Johnson et al. 2009), maybe all of them ${ }^{3}$. This is at first surprising as one can expect the most massive planets are the ones for which exciting the inclination is more difficult. If indeed such misalignments are frequent for the high-mass-planet population, this suggests a different evolution scenario for them. Maybe the more massive planets could not really slowly migrate because of the interactions with the disk. In that case, only more severe interactions with another planet or a star could be the cause of migration for massive planets, such interactions also affecting the inclination of the orbit. We also note that the $\lambda$-angles seem lower for the massive planets than those of the misaligned planets with lower masses; this also suggests a different scenario for the processes able to modify the inclination of low- and largemass planets.

Rossiter-McLaughlin observations of other systems with transiting massive planets should be performed to confirm whether or not they are preferentially tilted.

\section{Conclusion}

We presented an observation of the 12-hour-long transit of the highly eccentric, 111.4-day-period exoplanet HD 80606b performed in 2010 January. The transit light curve we present is among the first carried with the post-cryogenic Spitzer. Its shows systematic effects stronger than those seen in the Cold-Spitzer, but the accuracy remains clearly better than ground-based observations. Together with the SOPHIE measurements acquired at the same epoch, this is one of the first observational campaigns performed simultaneously in radial velocities and space-based high-accuracy photometry. With previously available datasets, this allows the parameters of this system to be significantly refined thanks to combined fits. There is a possible detection of a variation in the transit timing, which has to be confirmed by additional observations of HD $80606 \mathrm{~b}$ transits from ground or space. A dark spot was also possibly detected on the surface of this inactive star. The spin-orbit misalignment is clearly confirmed and the $\lambda$ angle is accurately measured. Like most of the massive planets for which this angle is measured, the orbit of HD $80606 \mathrm{~b}$ is misaligned with the equatorial plan of its host star. This suggests a separate evolution scenario for massive planets compared with Jupiter-mass planets.

Acknowledgements. We thank the Haute-Provence Observatory staff that allowed SOPHIE observations despite the ongoing break of the $193-\mathrm{cm}$ telescope, and in particular Fabien Fillion, François Moreau, Jacky Taupenas, Jean-Pierre Troncin and Stéphane Favard. We are grateful to C. Adami, D. Russeil and M. Dennefeld for their flexibility in the OHP $120-\mathrm{cm}$ photometry observations, M. Gillon and J. N. Winn for useful discussions, and the referee G. Laughlin. We thank the Spitzer director for having awarded us discretionary time, and the Spitzer staff and in particular Nancy Silbermann for their assistance in the preparation of the observation. We acknowledge support of the "Programme National de Planétologie" (PNP) of CNRS/INSU, the Swiss National Science Foundation, and the French National Research Agency (ANR-08-JCJC-0102-01 and ANRNT05-4-44463). D.E. is supported by CNES. A.E. is supported by a fellowship for advanced researchers from the Swiss National Science Foundation. N.C.S. acknowledges the support of the European Research Council/European Community under the FP7 through a Starting Grant, and the Fundação para a

3 The only exception, HATP-P-2, could actually be also misaligned as the inclination of the stellar rotation axis is unknown.
Ciência e a Tecnologia (FCT), Portugal, for a Ciência 2007 contract funded by FCT/MCTES (Portugal) and POPH/FSE (EC), and in the form of grants reference PTDC/CTE-AST/66643/2006 and PTDC/CTE-AST/098528/2008.

\section{References}

Agol, E., Steffen, J., Sari, R., \& Clarkson, W. 2005, MNRAS, 359, 567

Anderson, D. R., Hellier, C., Gillon, M., et al. 2010, ApJ, 709, 159

Baranne, A., Queloz, D., Mayor, M., et al. 1994, A\&AS, 119, 373

Barbieri, M., Alonso, R., Desidera, S., et al. 2009, A\&A, 503, 601

Boisse, I., Moutou, C., Vidal-Madjar, A., et al. 2009, A\&A, 495, 959

Boisse, I., Bouchy, F., Chazelas, B., et al. 2010a, New technologies for probing the diversity of brown dwarfs and exoplanets, EPJ Web of Conferences, in press [arXiv: 1001.0794$]$

Boisse, I., Eggenberger, A., Santos, N. C., et al. 2010b, A\&A, accepted

Bouchy, F., Pont, F., Melo, C., et al. 2005, A\&A, 431, 1105

Bouchy, F., Queloz, D., Deleuil, M., et al. 2008, A\&A, 482, L25

Bouchy, F., Hébrard, G., Udry, S., et al. 2009, A\&A, 505, 853

Chambers, J. E. 1999, MNRAS, 304, 793

Charbonneau, D., Alle, L. E., Megeath, S. T., et al. 2005, ApJ, 626, 523

Cochran, W. D., Redfield, S., Endl, M., \& Cochran, A. L. 2008, ApJ, 683, L59

Collier Cameron, A., Bruce, V. A., Miller, G. R. M., Triaud, A. H. M. J., \& Queloz, D. 2010, MNRAS, 403, 151

Deeg, H. J., Moutou, C., Erikson, A., et al. 2010, Nature, 464, 384

Deming, D., Seager, S., Richardson, L. J., \& Harrington, J. 2005, Nature, 434, 740

Désert, J.-M., Lecavelier des Etangs, A., Hébrard, G., et al. 2009, ApJ, 699, 478

Désert, J.-M., Sing, D. K., Vidal-Madjar, A., et al. 2010, ApJ, submitted

Ehrenreich, D., Hébrard, G., Lecavelier des Etangs, A., et al. 2007, ApJ, 668, L179

Fabrycky, D. C., \& Winn, J. N. 2009, ApJ, 696, 1230

Fazio, G. G., Hora, J. L., Allen, L. E., et al. 2004, ApJS, 154, 10

Fernandes, J., \& Santos, N. C. 2004, A\&A, 427, 607

Fossey, S. J., Waldmann, I. P., \& Kipping, D. M. 2009, MNRAS, 396, L16

Garcia-Melendo, E., \& McCullough, P. R. 2009, ApJ, 698, 558

Gillon, M. 2009, MNRAS, submitted [arXiv:0906.4904]

Gillon, M., Demory, B.-O., Barman, T., et al. 2007, A\&A, 471, L51

Gillon, M., Anderson, D. R., Triaud, A. H. M. J., et al. 2009, A\&A, 501, 785

Hébrard, G., Lemoine, M., Vidal-Madjar, A., et al. 2002, ApJ, 140, 103

Hébrard, G., Bouchy, F., Pont, F., et al. 2008, A\&A, 481, 52

Hébrard, G., Udry, S., Lo Curto, G., et al. 2010a, A\&A, 512, A46

Hébrard, G., Bonfils, X., Ségransan, D., et al. 2010b, A\&A, 513, 69

Hidas, M. G., Tsapras, Y., Street, R. A., et al. 2010, MNRAS, accepted [arXiv: 1002.1052 ]

Hirano, T., Suto, Y., Taruya, A., et al. 2010, ApJ, 709, 458

Holman, M. J., \& Murray, N. W. 2005, Science, 307, 1288

Jenkins, J. M., Borucki, W., J., Koch, D. G., et al. 2010, ApJ, submitted [arXiv: 1001.0416]

Johnson, J. A., Winn, J. N., Narita, N., et al. 2008, ApJ, 686, 649

Johnson, J. A., Winn, J. N., Albrecht, S., et al. 2009, PASP, 121, 1104

Kipping, D. M., \& Tinetti, G. 2010, MNRAS, accepted [arXiv: 0912.1133]

Knutson, H. A., Charbonneau, D., Allen, L. E., et al. 2007, Nature, 447, 183

Kurucz, R. L. 1979, ApJS, 40, 1

Laughlin, G., Deming, D., Langton, J., et al. 2009, Nature, 457, 562

Lecavelier des Etangs, A., Pont, F., Vidal-Madjar, A., \& Sing, D. 2008a, A\&A, 481, L83

Lecavelier des Etangs, A., Vidal-Madjar, A., Désert, J.-M., \& Sing, D. 2008b, A\&A, 485, 865

Loeillet, B., Shporer, A., Bouchy, F., et al. 2008, A\&A, 481, 529

Mamajek, E. E., \& Hillenbrand, L. A. 2008, ApJ, 687, 1264

Mandel, K., \& Agol, E. 2002, ApJ, 580, L171

Morales-Calderón, M., Stauffer, J. R., Kirkpatrick, J. D., et al. 2006, ApJ, 653,1454

Moutou, C., Pont, F., Bouchy, F., \& Mayor, M. 2004, A\&A, 424, L31

Moutou, C., Hébrard, G., Bouchy, F., et al. 2009, A\&A, 498, L5

Naef, D., Latham, D. W., Mayor, M., et al. 2001, A\&A, 375, L27

Narita, N., Enya, K., Sato, B., et al. 2007, PASJ, 59, 763

Narita, N., Sato, B., Hirano, T., \& Tamura, M. 2009a, PASJ, 61, L35

Narita, N., Hirano, T., Sato, B., et al. 2009b, PASJ, 61, 99

Narita,N., Sato, B., Hirano, T. et al. 2010, PASJ, 62, 653

Nesvorny, D., \& Beaugé, C. 2010, ApJ, 709, L44

Noyes, R. W., Hartmann, L. W., Baliunas, S. L., Duncan, D. K., \& Vaughan, A. H. 1984, ApJ, 279, 763 
A\&A 516, A95 (2010)

Ohta, Y., Taruya, A, \& Suto, Y. 2005, ApJ, 622, 1118

O'Toole, S. J., Tinney, C. G., Jones, H. R. A., et al. 2009, MNRAS, 392, 641

Pepe, F., Mayor, M., Galland, F., et al. 2002, A\&A, 388, 632

Perruchot, S., Kohler, D., Bouchy, F., et al., 2008, in Ground-based and Airborn Instrumentation for Astronomy II, ed. I. S., McLean, \& M. M., Casali, Proc. SPIE, 7014, 70140J

Pont, F., Gilliland, R. L., Moutou, C., et al. 2007, A\&A, 476, 1347

Pont, F., Knutson, H., Gilliland, R. L., Moutou, C., \& Charbonneau, D. 2008, MNRAS, 385, 109

Pont, F., Hébrard, G., Irwin, J. M., et al. 2009, A\&A, 509, 695

Pont, F., Endl, M., Cochran, W. D., et al. 2010, MNRAS, 402, L1

Queloz, D., Eggenberger, A., Mayor, M., et al. 2000, A\&A, 359, L13

Queloz, D., Bouchy, F., Moutou, C., et al. 2009, A\&A, 506, 303

Rabus, M., Alonso, R., Belmonte, J. A., et al. 2009, A\&A, 494, 391

Reach, W. T., Surace, J. A., Glaccum, W. J., et al. 2006, Infrared Array Camera Data Handbook, Version 3.0

Saar, S. H., \& Donahue, R. A. 1997, ApJ, 485, 741

Santos, N. C., Mayor, M., Naef, D., et al. 2000, A\&A, 361, 265

Scoupe et al. 2006, LPQ, 1931, 2
Sing, D. K. 2010, A\&A, 510, 21

Simpson, E. K., Pollacco, D., Hébrard, G., et al. 2010, MNRAS, 405, 1867

Triaud, A. H. M. J., Queloz, D., Bouchy, F., et al. 2009, A\&A, 506, 377

Tripathi, A., Winn, J. N., Johnson, J. A., et al. 2010, ApJ, 715, 421

Valenti, J. A., \& Fischer, D. A., 2005, ApJS, 159, 141

Winn, J. N. 2010, [arXiv: 1001.2010]

Winn, J. N., Noyes, R. W., Holman, M. J., et al. 2005, ApJ, 631, 1215

Winn, J. N., Johnson, J. A., Marcy, G. W., et al. 2006, ApJ, 653, L69

Winn, J. N., Johnson, J. A., Peek, K. M. G., et al. 2007, ApJ, 665, L167

Winn, J. N., Johnson, J. A., Narita, N., et al. 2008, ApJ, 682, 1283

Winn, J. N., Howard, A. W., Johnson, J. A., et al. 2009a, ApJ, 703, 2091

Winn, J. N., Johnson, J. A., Fabrycky, D., et al. 2009b, ApJ, 700, 302

Winn, J. N., Johnson, J. A., Albrecht, S., et al. 2009c, ApJ, 703, L99

Winn, J. N., Johnson, J. A., Howard, A. W., et al. 2010, ApJ, 718, 575

Wittenmyer, R. A., Welsh, W. F., Orosz, J. A., et al. 2005, ApJ, 632, 1157

Wittenmyer, R. A., Endl, M., Cochran, W. D., Levison, H. F., \& Henry, G. W. 2009, ApJS, 182, 97

Wolf, A. S., Laughlin, G., Henry, G. W., et al. 2007, ApJ, 667, 549

Wu, Y., \& Murray, N. 2003, ApJ, 589, 605 